\title{
COTERMINAL FAMILIES AND THE STRONG MARKOV PROPERTY
}

\author{
BY
}

\author{
A. O. PITTENGER AND C. T. SHIH( 1 )
}

ABSTRACT. Let $E_{\Delta}$ be a compact metric space and assume that a strong Markov process $X$ is defined on $E_{\Delta}$. Under the assumption that $X$ has right continuous paths with left limits, it is shown that a version of the strong Markov property extends to coterminal families, a class of random times which can be visualized as last exit times before $t$ from a fixed subset of $E_{\Delta}$. Since the random times are not Markov times, the conditioning $\sigma_{-f i e l d}$ and the new conditional probabilities must be defined. If $X$ is also assumed to be nearly quasileft continuous, i.e. branching points are permitted, two different conditionings are possible-one on the "past" of the random time and one on the "past plus present"-and two different conditional probabilities must be defined.

1. Introduction. The purpose of this paper is to prove a version of the strong Markov property for a class of random times resembling last exit times from sets before constant times $t>0$. To motivate the problem assume that $\left(\Omega, X_{t}, \theta_{t}, \mathcal{F}, \mathcal{F}_{t}, P^{x}\right)$ is a strong Markov process $w$ ith initial distribution $\mu$ and that $T$ is a stopping time. Then on $\{T<t\}$ the strong Markov property can be written as

$$
\left.P^{\mu}\left(X_{t} \in A \mid \mathcal{F}_{T}\right)=P^{X} T_{t-T} \in A\right) \text { a.s. } P^{\mu},
$$

where $\mathcal{F}_{T}$ is the usual $\sigma$-field of information up through $T$.(2) We are interested in finding an analogue of (1.1) with $T$ replaced by random times such as $L^{t}$, the last exit before $(t+0)$ from a given set, and $\mathcal{F}_{T}$ replaced by an appropriate $\sigma$-field. Since the conditioning now involves the future of the process, the distributions on the right of (1.1) must be altered, but we would hope that the dependence on only $X_{L^{t}}$ (or $X_{L^{t}}$ ) and $t-L^{t}$ could be maintained.

Presented to the Society, January 17, 1972; received by the editors March 3, 1972 and, in revised form, December $11,1972$.

AMS (MOS) subject classifications (1970). Primary 60 J25; 60 J35, 60 J 40.

Key words and phrases. Markov process, strong Markov property, Hunt process, Standard process, last exit time, coterminal time.

(1) The research of both authors was supported in part by NSF Grant GP.28877.

(2) The right-hand side of the equation means $P^{X_{T}(\omega)}\left(\omega^{\prime}: X_{t-T(\omega)}\left(\omega^{\prime}\right) \epsilon A\right)$, or $P_{t-T}\left(X_{T}, A\right)$ where $P_{s}(x, A)$ denotes the transition function. Similar expressions will appear repeatedly throughout the paper, but usually we will omit the $\omega$ and exhibit $\omega^{\prime}$ only when confusion might otherwise arise. 
Let us give two examples of the type of problem we have in mind. First suppose the given Markov process is reflecting Brownian motion on $[0, \infty)$, $\mu(d x)=\epsilon_{0}(d x), T$ is the first hit of $\{0\}$ and $L^{t}$ the last hit of $\{0\}$ prior to $t$. Then a straightforward computation shows that a.s. on $\left\{L^{t}<t\right\}$

$$
E^{0}\left[f\left(x_{t}\right) \mid \sigma\left(L^{t}\right)\right]=\int_{0}^{\infty} Q\left(t-L^{t}, d y\right) f(y),
$$

where $Q(s, d y)=\left(\mathrm{y} \exp \left(-y^{2} / 2 s\right) / s\right) d y$. Moreover, if $H_{t}(x, f)=E^{x}\left[f\left(X_{t}\right) ; T>t\right]$, then

$$
\int s^{-1 / 2} Q(s, d x) H_{t}(x, f)=\int(s+t)^{-1 / 2} Q(s+t, d y) f(y),
$$

i.e. $\left\{s^{-1 / 2} Q(s, d y), 0<s<\infty\right\}$ is an entrance law relative to $H_{t}$.

For a somewhat less familiar example let $P(t)=\left(\left(p_{i j}(t)\right)\right)$ be a transition matrix on a countable state space $E$ and let $\hat{E}$ denote the Doob-Ray compactification of $E$ [4]. Then there is a strong Markov process on $\hat{E}$ which has $P(t)$ for its transition matrix and which is "nearly quasi-left continuous" as defined in $\$ 2$ below. Choose $B$ to be any finite subset of $E$ with $b \in B$. Define $L^{t}$ to be the last hit of $B$ before $(t+0)$, and let $F(t)=\left(\left(f_{i j}(t)\right)\right)$ with $f_{i j}(t)=$ $P^{i}\left(X_{t}=j, T_{B}>t\right)$. Then it can be shown [8] that there exists a family of entrance laws $\{\eta(c, t, j) ; c \in B, 0<t<\infty\}$ relative to $F(t)$ such that for $0<s<t$

$$
\eta(c, t-s, j) / \eta(c, t-s)=P\left[X_{t}=j \mid L^{t}=s, X_{L^{t}-}=c\right] \text { a.s. } P^{\mu}
$$

where $\eta(c, t-s)=\Sigma_{j \in E-B} \eta(c, t-s, j)$.

The fact that there are analogous results for such disparate processes provides a strong motivation for attempting to prove similar results for general strong Markov processes on general state spaces, and that is what is done in this paper. To summarize more precisely, given a suitable family $\left\{L^{t}, t>0\right\}$ of random times, a so-called coterminal family, and an arbitrary initial distribution $\mu$, there exist probability measures $D(x, s, \cdot)$ [and $Q(x, s, \cdot)]$ independent of $\mu$ and such that $D\left(X_{L^{t}}, t-L^{t},.\right)$ [or $\left.Q\left(X_{L^{t}}, t-L^{t}, \cdot\right)\right]$ determines the distribution of the process at $t$ in a manner analogous to (1.2). In addition for $x$ in an appropriate set $G_{D}\left(\right.$ or $\left.G_{Q}\right), D(x, .,$.$\left.) [or Q(x, .,).\right]$ can be normalized to define entrance laws relative to an appropriate transition probability as in (1.3). Moreover a past of $L^{t}$ can be defined and a version of the strong Markov property obtained: the evolution of the process from $L^{t}$ to $t$ is independent of the past of $L^{t}$, and depends only on $X_{L^{t}}$ (or $\left.X_{L^{t}}\right)$ and $L^{t}$ itself.

A basic motivation for some of our methods is the axiomatic approach to coterminal times developed in. [7], and in $\$ 3$ we borrow heavily from their work. Those familiar with [7] will find that Theorems 6 and 8 below represent an extension of [7, Theorem 3$]$ by establishing the conditional independence of the 
$L$-past and $L$-future and by giving an expression for the post- $L$ process in terms of $X_{L}$ or $X_{L_{-}}$. Although these results could have been obtained directly by the methods of $\$ \bar{\S}_{5}$ and 6 , we prove them by using the relationship $L=\lim _{t \mid \infty} L^{t}$.

The first part of this paper develops the necessary technical machinery. In $\$ 2$ we discuss the assumptions on the process, particularly the property of nearly quasi-left continuity. In $\$ 3$ the concept of a coterminal family is developed and its elementary properties derived. This treatment is patterned on [7], the main difference being the emphasis on $\left\{L^{t}, t>0\right\}$ as opposed to a coterminal time $L$. In $\$ 4$ we combine the definitions from [3] and [7] to define a "past" and a "past plus present" of an ordinary random variable. We do not pretend to a comprehensive discussion such as is given in [3], but rather derive only those results necessary for our purposes.

The bulk of the work of this paper is contained in $\$ \$ 5$ and 6 where the conditional distributions $D$ and $Q^{b}, b$ continuous, are defined and their basic probabilistic interpretation established.(3) We could emphasize here that the results of $\S 5$ do not require any form of quasi-left continuity nor do any subsequent resulț which involve $D$ exclusively. The requirement of nearly quasi-left continuity is vital only for the proof of the existence of $Q^{b}\left(X_{L^{t}-}, t-L^{t},.\right)$.

In $\$ 7$ we relate $D$ and $Q^{b}$ by means of an intermediary measure which describes the distribution of $X_{L^{t}}$ given the past. The fact that $D$ and $Q^{b}$ can be normalized to become entrance laws is established in $\$ 8$, and it is shown that a.s. on an appropriate set $\Omega_{t}$ both $X_{L^{t}}$ and $X_{L^{t}}$ - are associated with such an entrance law. This property is used in $\$ 9$ to establish versions of the strong Markov property at $L^{t}$. Moreover all of these results can be carried over to the coterminal time $L=\lim _{t \rightarrow \infty} L^{t}$, and this is verified in $\$ 10$.

In order to obtain these results we have had to be quite careful about establishing the existence of $Q^{b}(x, s,$.$) and D(x, s,$.$) . To prove that this is not a$ vacuous difficulty we give in $\S 11$ an example of a process and a coterminal family for which the conditional distributions $D(x, s,$.$) and Q^{b}(x, s,$.$) do not exist for$ certain $x$.

2. Notation and definition of the process. Let $\left(E_{\Delta}, \rho\right)$ be a compact metric space, $\Delta$ a distinguished point in $E_{\Delta}$, and let $\beta$ denote the $\sigma$-field of Borel sets and $\hat{\mathscr{B}}$ the universally measurable sets of $E_{\Delta}$. $\mathcal{C}, \mathcal{E}$, and $\hat{\mathcal{E}}$ will denote respectively the spaces of real, continuous functions of bounded $\mathscr{B}$ measurable functions and of bounded $\hat{\Re}$ measurable function on $E_{\Delta}$.

We shall consider a strong Markov process $X=\left(\Omega, X_{t}, \mathcal{F}, \mathcal{F}, \theta_{t}, P^{x}\right)$ on $E_{\Delta}$ such that the paths $X_{t}$ are right continuous and have left limits and such that $\Delta$ is an absorbing point: $X(t)=\Delta$ implies $X(s+t)=\Delta$ for all $s>0$. In general our

(3) In the motivation above, we were using $Q=Q^{1}$. 
notation follows that of [1]: $\mathcal{F}$ is the intersection of all completions of the minimal $\sigma$-field $\mathcal{F}^{0}=\sigma\left(X_{t}, t>0\right)$ with respect to the measures $P^{\mu}()=.\int \mu(d x) P^{x}(),. \mu$ a probability on $\left(E_{\Delta}, \rho\right)$, and $\mathcal{F}_{t}$ is the completion of $\mathcal{F}_{t}^{0}=\sigma\left(X_{s}, s \leq t\right)$ in $\mathcal{F}$. Implicit in the above is that for $\Lambda \in \mathcal{F}^{0}$, and thus for $\Lambda \in \mathcal{F}, P^{x}(\Lambda)$ is $\hat{B}$ measurable. We shall assume that the $\mathcal{F}_{t}$ are right continuous, i.e., $\mathcal{F}_{t}=\mathcal{F}_{t^{+}}=$ $\bigcap_{u>t} \mathcal{F}_{u}$. The operator $\theta_{t}$ stands for the usual shift operator on the sample space $\Omega$ satisfying $X_{s}\left(\theta_{t} \omega\right)=X_{s+t}(\omega)$. A stopping time $T$ is relative to $\mathcal{F}_{t}$, i.e. a function from $\Omega$ to $[0, \infty]$ with $\{T<t\} \in \mathcal{F}_{t}$ for all $t$, and $\mathcal{F}_{T}$ denotes the $\sigma$-field of sets $\Lambda$ in $\mathcal{F}$ with $\Lambda \cap\{T<t\} \in \mathcal{F}_{t}$ for all $t$. In one of several equivalent forms the strong Markov property then says that for any initial probability $\mu$ and stopping time $T$

$$
E^{\mu}\left[\phi\left(\theta_{T}\right) \mid \mathcal{F}_{T}\right]=E^{X} T(\phi) \text { a.s. } P^{\mu}
$$

on $\{T<\infty\}$ for any bounded $\mathcal{F}$ measurable function $\phi$.

In this paper we deviate somewhat from [1] by allowing the possible existence of branching points, namely those $x$ with $P^{x}\left(X_{0}=x\right)<1$, which by the Blumenthal 0-1 law is equivalent to $P^{x}\left(X_{0}=x\right)=0$. We shall let $\nu(x, d y)$ denote the mea sure $P^{x}\left(X_{0} \in d y\right)$ for branching points and also for ordinary (nonbranching) points for which $\nu(x, d y)$ is then the unit mass at $x$. Note that $\nu(x, B)$ is $\hat{\mathscr{B}}$ measurable for $B \in \hat{\mathfrak{B}}$. It follows that the set of branching points $E_{b}$ is universally measurable. (We shall also denote $E_{\Delta}-E_{b}$ by $E_{r}$.)

Although we do not need it in this paper, it would not be unreasonable to assume that $E_{b}$ is nearly Borel measurable; that is, for any $\mu$ there exist Borel sets $B_{1}$ and $B_{2}$ with $B_{1} \subset E_{b} \subset B_{2}$ and $P^{\mu}\left(X_{t} \in B_{2}-B_{1}\right.$ for some $\left.t\right)=0$. Since the paths are right continuous, it is known (see [6]) that if $B$ is nearly Borel then for any $\mu$ there exist increasing compact subsets $F_{n}$ of $B$ such that the first hitting times $T_{F_{n}}=\inf \left\{t>0: X_{t} \in F_{n}\right\}$ decrease to $T_{B}$ a.s. $P^{\mu}$. From this and the strong Markov property the above assumption implies that for almost all paths $E_{b}$ is never visited. In this paper, however, we only need the fact that if $R$ is any random time, $\left\{X(R) \in E_{b}, R<\infty\right\}$ has $P^{\mu}$ measure zero for any $\mu$, and that requires only $E_{b} \in \hat{B}$ since $P\left[X(R) \in E_{b}, R<\infty\right]>0$ implies that, for some compact subset $F \subset E_{b}, P^{\mu}\left[T_{F}<\infty\right]>0$, which is impossible by the strong Markov property.

Theorem 1 and subsequent results based only on Theorem 1 hold for a strong Markov process as defined above. However, we do need a type of quasi-left continuity for Theorem 2, and to emphasize the fact that this property is to be consistent with the existence of branching points we shall call it nearly quasi-left continuity, although the word "nearly" could be safely suppressed. Specifically, if $T_{n} \uparrow T$ are stopping times then for any $\mu$ and any $f \in \mathcal{E}$ a.s. $P^{\mu}$ 


$$
E^{\mu}\left[f\left(X_{T}\right) \mid \bigvee_{n} \mathcal{F}_{T_{n}}\right]=\int \nu\left(X_{T-}, d y\right) f(y)
$$

on $\left\{T_{n}<T<\infty\right.$, all $\left.n\right\}$. Equivalently if $\phi$ is a bounded $\mathcal{F}$-measurable function, a.s. $P^{\mu}$ on $\left\{T_{n}<T<\infty\right.$, all $\left.n\right\}$,

$$
E^{\mu}\left[\phi\left(\theta_{T}\right) \mid \bigvee_{n} \mathcal{F}_{T_{n}}\right]=\int \nu\left(X_{T-}, d y\right) E^{y}(\phi) .
$$

In both equations $\bigvee_{n} \mathcal{F}_{T_{n}}$ stands for the minimal $\sigma$-field containing all of the $\mathcal{F}_{T_{n}}$. Note that this implies a.s. $P^{\mu}$

$$
X\left(T_{n}\right) \rightarrow X(T)
$$

on $\left\{T_{n}<T<\infty\right.$, all $\left.n ; X\left(T_{-}\right) \in E_{r}\right\}$, where $E_{r}=E_{\Delta}-E_{b}$. Thus if $E_{b}=\varnothing$, the process $X$ must be a Hunt process. (4) If the process is standard instead of Hunt, there will be a slight and obvious modification in the statements of Theorem 2 and the results based on it. (See the remark following the statement of Theorem 2.)

We should point out that the assumption of nearly quasi-left continuity is not particularly restrictive. In fact [10] shows that if $X$ is a strong Markov process on a "nice" state space and which satisfies a rather weak condition, then by enlarging the state space $X$ can be assumed to satisfy the above quasi-left continuity, where now $X_{t-}$ need not be a point in the original state space (but will then be a branching point). For additional comments on this topic see [6].

3. Coterminal families of random times. In this section we define precisely the random times with which we will be concerned in the remainder of the paper. Following Meyer, Smythe and Walsh [7] our approach is completely axiomatic, and it may help if the reader interprets the definitions and results of this section in terms of last exit times.

We assume given killing operators $k_{t}: \Omega \rightarrow \Omega$ satisfying $X_{s} \circ k_{t}=X_{s}$ for $s<t$ and $=\Delta$ for $s \geq t,(5)$ and observe that $k_{t}^{-1}(\Lambda) \in \mathcal{F}_{t}^{0}$ for $\Lambda \in \mathcal{F}^{0}$, where $\mathcal{F}^{0}$ and $\mathcal{F}_{t}^{0}$ are the minimal fields generated by the process. By an exact coterminal time we will mean an $\mathcal{F}$ measurable function $L: \Omega \rightarrow[0, \infty]$ satisfying the axioms

(4) More precisely, $X$ satisfies all the properties of a Hunt process except that the semigroup need not be Borel measurable. Future references to Hunt and standard processes should be interpreted similarly.

(5) As with $\theta_{t}$, the assumption about the existence of $k_{t}$ on $\boldsymbol{\Omega}$ is a matter of convenience. Also, the reader may take $\Omega$ to be the space of all right continuous functions $\omega:[0, \infty) \rightarrow E_{\Delta}$ with left limits; in this case $\theta_{t}$ and $k_{t}$ are uniquely defined. 
(i) $L \circ k_{s}$ is $\mathcal{F}_{s}$ measurable,

(ii) $L \circ \theta_{s}=(L-s)^{+}=(L-s) \vee 0$,

(iii) $L \circ k_{s}=L$ on $\{L<s\}$,

(iv) $L \circ k_{s} \leq s$ for all $s$, and

(v) $L=\lim _{s \mid \infty} L \circ k_{s}$.

Now suppose that $\left\{L^{t}, t \geq 0\right\}$ is defined by

$$
L^{t}=\lim _{u\lfloor t} L \circ k_{u} .
$$

Then it can be shown that $\left\{L^{t}\right\}$ has the properties

(1) $L^{t}$ is $\mathcal{F}_{t}$ measurable,

(2) $0 \leq L^{t} \circ k_{t} \leq L^{t} \leq t$

(3) $L^{t-s} \circ \theta_{s}=\left(L^{t}-s\right)^{+}$for $s<t$,

(4) $\lim _{u\rfloor s} L^{t} \circ k_{u}=L^{s}$ for $s<t$, and

(5) if $L^{t}<s<t$, then $L^{t}=L^{s}$.

For example if $L$ is the last exit from a set $A$ before $\zeta=\inf \left\{u: X_{u}=\Delta\right\}$, then $L^{t}$ will be the last exit from $A$ before $(t+0)$.

Our primary concern here is not with $L$ but rather with $\left\{L^{t}\right\}$, and we give as the basic definition

Definition 3.1. A family $\left\{L^{t}, t \geq 0\right\}$ of $\mathcal{F}$ measurable functions is a coterminal family if it satisfies (1) through (5).

In practice of course the axioms would hold with exceptional sets depending on $s$ and $t$, and the best we could hope for would be a set of full measure on which (1) through (5) hold for all $s$ and $t$. To avoid this technicality we simply assume the axioms are valid on all of $\Omega$. Note that whatever the set $\Omega$ of definition, we must have $k_{t} \Omega \subset \Omega$ even as $\theta_{t} \Omega \subset \Omega$ for the shift operator.

In [7] the axioms for a coterminal time were used to define an associated terminal time $T$, and then these concepts were applied to prove that the post$L$ process is a strong Markov process. We will use the coterminal family to define $T$ and to derive some of the ir results without reference to $L$. Since the proofs are short, we include them here rather than referring to [7].

Lemma 3.1. A coterminal family $\left\{L^{t}, t \geq 0\right\}$ bas the following properties:

(a) $L^{s} \leq L^{t}$ for $s<t$.

(b) If $0=L^{s}<L^{t}$, then $L^{t} \geq s$.

(c) If $r<s<t$, then $L^{r}=L^{r} \circ k_{s} \leq L^{s} \circ k_{s}=L^{t} \circ k_{s} \leq L^{t}$.

(d) $L^{t} \circ k_{r} \leq L^{t} \circ k_{s}$ for $r<s$.

(e) $L^{t} \omega_{\Delta}=0$, where $X_{s}\left(\omega_{\Delta}\right)=\Delta$ for all $s \geq 0$.

(f) $\lim _{t ! s} L^{t}=L^{s}$. 
Proof. The first two assertions follow easily from the definition. The first equality in (c) follows from

$$
L^{r} \circ k_{s}=\lim _{\epsilon \downarrow 0}\left(L^{s} \circ k_{r+\epsilon}\right) \circ k_{s}=\lim _{\epsilon \downarrow 0} L^{s} \circ k_{r+\epsilon}=L^{r},
$$

while the last two relations follow from

$$
L^{t} \circ k_{s}=\lim _{\epsilon ! 0} L^{t} \circ k_{s+\epsilon} \circ k_{s}=L^{s} \circ k_{s} \leq L^{s} \leq L^{t} .
$$

(d) then follows from (c), and (e) is a consequence of (2). Finally we need only consider the case $L^{s}<s$ to prove right continuity. By (4), $L^{t} \circ k_{s+2 \epsilon}<s$ for small $\epsilon>0$ and hence $L^{s+\epsilon}=L^{s+\epsilon} \circ k_{s+2 \epsilon}<s$ or $L^{s+\epsilon}=L^{s}$.

A key definition is that of the associated terminal time.

Definition 3.2. The terminal time associated with $\left\{L^{t}\right\}$ is $T \equiv \inf \left\{t: L^{t}>0\right\}$.

To see that $T$ is in fact a terminal time and to list its basic properties we have

Lemma 3.2. (a) $T$ is a perfect, exact terminal time.

(b) $T=\infty$ iff $L^{t}=0$ for all $t$. If $T<t, T \leq L^{t}$.

(c) Let $s<t$. Then $T \circ \theta_{s} \circ k_{t}=\infty$ iff $L^{t} \circ k_{t} \leq s$.

(d) If $L^{t}<t, T \circ \theta_{t}>0$.

(e) Let $b<t$. Then $L^{t}<b$ iff $L^{b}<b$ and $T \circ \theta_{b}>t-b$.

(f) Let $a<b<t$. Then $a<L^{t} \leq b$ iff $T \circ \theta_{a} \leq b-a$ and $T \circ \theta_{b}>t-b$.

Proof. (a) From (3)

$$
T \circ \theta_{t}=-t+\inf \left\{u: L^{u}>t\right\}
$$

If $T>t+\epsilon, L^{u}=0$ for $u \leq t+\epsilon$, and by Lemma 3.1 (b)

$$
\inf \left\{u: L^{u}>t\right\}=\inf \left\{u: L^{u}>0\right\}
$$

i.e. $T=t+T \circ \theta_{t}$ on $\{T>t\}$. Since

$$
\{T<t\}=\bigcup_{r<t}\left\{L^{r}>0\right\} \in \mathcal{F}_{t}, \quad r \text { rational, }
$$

$T$ is a perfect terminal time. To prove the property of exactness, $T=$ $\lim _{t ! 0}\left(t+T \circ \theta_{t}\right)$ on all of $\Omega$, it suffices to check the set $\{T=0\}$, and that is immediate from (3.2).

(b) The first sentence is by definition. For the second suppose $0<L^{t}<t$. Then $L^{t}<s$ implies $L^{s}>0, T \leq s$ and hence $T \leq L^{t}$.

(c) Use equation (3.2) and Lemma 3.1 (c).

(d) If $T \circ \theta_{t}=0$, then $L^{\epsilon} \circ \theta_{t}>0$ for all $\epsilon>0$. Hence $L^{t+\epsilon}>t$ by (3), and $L^{t}=t$ by right continuity.

(e) If $L^{t}<b, L^{b}<b$ is immediate, and $T \circ \theta_{b}>t-b$ follows from (3.2) and right continuity. Conversely $L^{t} \leq b$ follows from $t-b<T \circ \theta_{b}$ and the strict inequality from (5): 


$$
L^{t}=\lim _{\epsilon \rightarrow 0} L^{b+\epsilon}=L^{b}<b .
$$

(f) If- $a<L^{t} \leq b, L^{b+\epsilon-a} \circ \theta_{a}>0$ and $T \circ \theta_{a} \leq b-a$ while $t-b<t \circ \theta_{b}$ follows as in (e) by noting that $L^{t}=L^{t+\epsilon}$ for small $\epsilon>0$. Conversely $T \circ \theta_{a} \leq$ $b-a$ implies $L^{b+\epsilon-a} \circ \theta_{a}>0, L^{b+\epsilon}>a$ and hence $L^{t}>a$. From $T \circ \theta_{b}>t-b$, $L^{t} \leq b$ follows as in (e).

It is an easy matter to verify that if $s<T$, then $T \circ k_{s}=\infty$ while if $s>T$, $T \circ k_{s}=T$. In [7] it is shown that the correspondence between such terminal times and exact coterminal times is one-to-one, and the correspondence carries over to coterminal families using the facts that

$$
L=\lim _{t \rightarrow \infty} L^{t}
$$

is an exact coterminal time and that $L^{t}$ can be recovered from $L$ via (3.1). Consequently, a coterminal family is not merely an artifice, but rather is another way of looking at a terminal time.

We will not pursue these ideas further but we will include the derivation of $\left\{L^{t}\right\}$ from its associated $T$.

Lemma 3.3. For all $t>0$ define

$$
\bar{L}^{t}=\lim _{u\lfloor t} \sup \left\{v: T \circ \theta_{v} \circ k_{u}<\infty\right\}
$$

Then $\bar{L}^{t}=L^{t}$.

Proof. Suppose $t<v<u<w$ and $T \circ \theta_{v} \circ k_{u}<\infty$. Then for some $s_{0}>u$, $L^{s} 0 \circ k_{u}>v$ by equation (3.2). But then, for $s>\max \left(w, s_{0}\right), L^{s} \circ k_{w}>v$, hence $T \circ \theta_{v} \circ k_{w}<\infty$, and it follows that the limit in the definition of $\bar{L}^{t}$ exists. Now suppose that for some $s \bar{L}^{t}<s$. Then $T \circ \theta_{s} \circ k_{u}=\infty$ for $u$ close to $t$, hence $L^{t}=L^{t} \circ k_{u} \leq s$ and $L^{t} \leq \bar{L}^{t}$. On the other hand, if $L^{t}<s<\bar{L}^{t}, T \circ \theta_{s} \circ k_{u}<\infty$ for all $u>t$ and for some $s<a \leq u$

$$
s<L^{a} \circ k_{u} \leq L^{u} \circ k_{u} \leq L^{u} \text {. }
$$

Since $L^{u} \downarrow L^{t}$, this contradicts $L^{t}<s$ and proves the lemma.

4. The past of a random time. The definition of the $\sigma$-field representing the past of $L^{t}$ is crucial in the proof of the strong Markov property. In this section we give the appropriate definitions and derive a number of elementary results which will be used in the sequel. Our approach follows closely that of Chung and Doob [3], where a more complete discussion appears, but we also incorporate a definition from [7] which is the natural generalization of the usual $\sigma$-field associated with a stopping time. In Lemma 4.3 we show when that field corresponds to one defined in [3]. Again for the sake of completeness, proofs are included.

Definition 4.1. Let $R$ be a random time. By the past of $R$ we will mean 


$$
\mathcal{F}(R-)=\sigma\left\{F_{t} \cap\{t<R\}, 0 \leq t\right\}
$$

i.e. the $\sigma$-field generated by sets $\Lambda_{t} \cap\{t<R\}$ with $\Lambda_{t} \in \mathcal{F}_{t}$. Further, define

$$
\mathcal{F}^{*}(R)=\sigma\left\{\mathcal{F}_{t} \cap\{t \leq R\}, 0 \leq t\right\}
$$

and

$$
\mathfrak{F}^{* *}(R)=\mathscr{F}\left(R_{-}\right) \vee \sigma\left(X_{R}\right) .
$$

By the past plus present we will mean

$$
\begin{aligned}
& \mathcal{F}(R)=\text { the } \sigma \text {-field generated by } \mathcal{F}\left(R_{-}\right) \text {and } \\
& \mathcal{F}_{S} \cap\{S=R\} \text { for all stopping times } S \text {. }
\end{aligned}
$$

Two $\sigma$-fields containing a bit of the future of $R$ are

$$
\mathcal{F}(R+)=\bigwedge_{\epsilon>0} \mathcal{F}((R+\epsilon)-)
$$

and

$$
\begin{array}{r}
\mathcal{F}(R(+))=\left\{\Lambda \in \mathcal{F}: \text { for all } u \geq 0 \text { there exists a } \Lambda_{u} \in \mathcal{F}_{u}\right. \\
\text { such that } \left.\Lambda \cap\{R<u\}=\Lambda_{u} \cap\{R<u\}\right\}
\end{array}
$$

Note that $\mathcal{F}(R(+))$ reduces to $\mathcal{F}_{R}$ in the event that $R$ is a stopping time. Further, the $\sigma$-field $\mathcal{F}^{*}(R)$ is defined in [3] and $\mathcal{F}^{* *}(R)$ is a natural generalization of $\mathcal{F}^{*}(R)$. Neither of the se fields plays a role in subsequent sections, but we do include them in Lemma 4.1 (c) for completeness.

Definition 4.2. $R$ will be called bonest if $R$ is $\mathcal{F}(R(+))$ measurable.

Note then that $L^{t}$ is honest since for $u<v$

$$
\left\{L^{t}<u\right\} \cap\left\{L^{t}<v\right\}=\left\{T \circ \theta_{u}>v-u\right\} \cap\left\{L^{t}<v\right\} .
$$

We first collect some preliminary results about $\mathfrak{F}(R-)$ and $\mathcal{F}(R)$.

Lemma 4.1. Let $R, S_{n}$ and $S$ be random times.

(a) If $R \leq S$ then $\mathcal{F}\left(R_{-}\right) \subset \mathcal{F}\left(S_{-}\right)$iff $R \in \mathcal{F}(S-)$.

(b) $\mathscr{F}(R-) \cap\{R=S\}=\mathscr{F}(S-) \cap\{R=S\}$.

(c) $\sigma(R) \subset \mathfrak{F}(R-) \subset \mathcal{F}^{*}(R) \subset \mathfrak{F}^{* *}(R) \subset \mathcal{F}(R)$.

(d) If $S_{n} \leq S=\lim S_{n}$ and $S_{n} \in \mathcal{F}(S-)$, then $\bigvee_{n} \mathcal{F}\left(S_{n}-\right)=\mathcal{F}(S-)$.

Proof. Since $\Omega \cap\{t<R\} \in \mathcal{F}(R-), \sigma(R) \subset \mathcal{F}(R-)$ and the necessity in (a) follows. Conversely if $R \in \mathcal{F}(S-)$

$$
\mathcal{F}_{t} \cap\{t<R\}=\mathcal{F}_{t} \cap\{t<S\} \cap\{t<R\} \in \mathcal{F}(S-) \text {. }
$$

Part (b) is immediate from the definition.

The first inclusion of (c) has already been shown while the second follows 
from the definition. For the third inclusion it suffices to show $\mathcal{F}_{u} \cap\{u=R\} \subset$ $\mathcal{F}^{* *}(R)$ for all $u$, and this reduces to showing that $\sigma\left(X_{v}\right) \cap\{u=R\} \subset \mathcal{F}^{* *}(R)$ for $v \leq u$. If $v=u$ this is certainly the case, while if $v<u$

$$
\sigma\left(X_{v}\right) \cap\{u=R\}=\sigma\left(X_{v}\right) \cap\{v<R\} \cap\{u=R\} \subset \mathcal{F}(R-) .
$$

For the last inclusion we need only show that $\sigma\left(X_{R}\right) \subset \mathcal{F}(R)$, and we do this by examining $\sigma\left(X_{R}\right) \cap\left\{X_{R} \neq X_{R-}, R<\infty\right\}$ and $\sigma\left(X_{R}\right) \cap\left\{X_{R}=X_{R_{-}}, R<\infty\right\}$. Let $R_{m n}$ denote the $n$th jumping time of magnitude greater than or equal to $1 / m$. Then $\left\{X_{R} \neq X_{R-}, R<\infty\right\}=\bigcup_{m, n}\left\{R=R_{m n}<\infty\right\}$ and

$$
\sigma\left(X_{R}\right) \cap\left\{R=R_{m n}<\infty\right\} \subset \mathcal{F}_{R_{m n}} \cap\left\{R=R_{m n}\right\} \subset \mathcal{F}(R) .
$$

Since $\sigma\left(X_{R}\right) \cap\left\{X_{R}=X_{R-}, R<\infty\right\}=\sigma\left(X_{R-}\right) \cap\left\{X_{R}=X_{R-}, R<\infty\right\}$ is also in $\mathcal{F}(R)$, the proof of (c) is complete. Finally, (d) is immediate from (a) and the definitions.

In the proofs of Theorems 1 ard 2 below it is convenient to work with $\mathcal{F}(S(+))$, and we record without proof some elementary properties.

Lemma 4.2. (a) If $R \leq S$, then $\mathcal{F}(R(+)) \subset \mathcal{F}(S(+))$.

(b) If $\{R<S\} \in \mathcal{F}(S(+))$, then $\mathcal{F}(R(+)) \cap\{R<S\} \subset \mathcal{F}(S(+))$.

(c) If $\lim _{n} S_{n}=S \leq S_{n}$, then $\mathcal{F}(S(+))=\bigwedge_{n} \mathcal{F}\left(S_{n}(+)\right)$.

(d) If $R \in \mathcal{F}(S-)$, then $\mathcal{F}(R(+)) \cap\{R<S\} \subset \mathcal{F}(S-)$.

For honest random variables the $\sigma$-fields with a bit of the future coincide:

Lemma 4.3. $\mathcal{F}(S+)=\mathcal{F}(S(+))$ iff $S$ is bonest.

Proof. The necessity follows from Lemma 4.1 (c). Conversely, if $S$ is honest and $t<u$

$$
\Lambda_{t} \cap\{t<S\} \cap\{S<u\}=\Lambda_{t} \cap\{t<S<u\} \cap\{S<u\}=\Gamma_{u} \cap\{S<u\}
$$

for some $\Gamma_{u} \in \mathcal{F}_{u}$; i.e. $\mathcal{F}(S-) \subset \mathcal{F}(S(+))$. Since $S$ is also $\mathcal{F}((S+\epsilon)+)$ measurable, $\mathcal{F}((S+\epsilon)-) \subset \mathcal{F}((S+\epsilon)(+))$ and $\mathcal{F}(S+) \subset \Lambda_{\epsilon>0} \mathfrak{F}((S+\epsilon)(+))=\mathfrak{F}(S(+))$. The proof is completed by noting that $\mathcal{F}(S(+)) \subset \mathcal{F}((S+\epsilon)-)$ from Lemma $4.2(\mathrm{~d})$.

Combining Lemma 4.3 with Lemma 4.2 (c) and (d), we have the useful result

Lemma 4.4. Suppose $S_{n} \in \mathcal{F}(S-)$ and is bonest. Then if $\Gamma=\left\{S_{n}<S=\right.$ $\lim _{m} S_{m}$, all $\left.n\right\}$

$$
\left(\bigvee_{n} \mathcal{F}\left(S_{n}(+)\right)\right) \cap \Gamma=\mathfrak{F}\left(S_{-}\right) \cap \Gamma .
$$

We will also require some knowledge of the relations between the $\sigma$-fields associated with random times $S$ and $T$ when $T$ is a stopping time.

Lemma 4.5. Suppose $T$ is a stopping time. Then 
(a) $\mathcal{F}(T(+)) \cap\{T<S\} \subset \mathcal{F}(S-)$.

(b) $\mathcal{F}(S(+)) \cap\{S \leq T\} \subset \mathcal{F}(T(+)) \cap\{S \leq T\}$.

Note that (b) bolds with $\{S \leq T\}$ replaced by $\{S=T\}$ or by $\{S<T\}$.

Proof. For (a) it suffices to show $\{T<S\} \in \mathcal{F}(S-)$, and that is immediate. For (b) let $T_{n}=k \cdot 2^{-n}$ on $\left\{(k-1) 2^{-n} \leq T<k \cdot 2^{-n}\right\}$ so that $\mathcal{F}(T(+))=$ $\Lambda_{n} \mathcal{F}\left(T_{n}(+)\right)$. If $\Lambda \in \mathcal{F}(S(+))$ with $\Lambda \cap\{S<u\}=\Lambda_{u} \cap\{S<u\}, \Lambda_{u} \in \mathcal{F}_{u}$, we may assume $\Lambda_{u} \subset \Lambda_{v}$ for dyadic rationals $u<v$. Letting $t_{k}=k \cdot 2^{-n}$ define $\Gamma_{n}=$ $\bigcup_{k} \Lambda_{t} \cap\left\{T_{n}=t_{k}\right\}$ so that $\Gamma_{n} \in \mathcal{F}\left(T_{n}(+)\right), \Lambda \cap\left\{S<T_{n}\right\}=\Gamma_{n} \cap\left\{S<T_{n}\right\}$, and $\Gamma_{n} \supset \Gamma_{n+1}^{k}$. Hence $\Gamma=\lim _{n} \Gamma_{n}$ is in $\mathcal{F}(T(+))$, and

$$
\begin{aligned}
\Gamma \cap\{S \leq T\} & =\lim _{n} \Gamma_{n} \dot{\cap}\left\{S<T_{n}\right\} \cap\{S \leq T\} \\
& =\lim _{n} \Lambda \cap\left\{S<T_{n}\right\} \cap\{S \leq T\}=\Lambda \cap\{S \leq T\} .
\end{aligned}
$$

The following result is not used in this paper but should prove useful in applications of our results.

Corollary. Suppose $R \leq S$. Then $\mathcal{F}(R) \subset \mathcal{F}(S)$ iff $R \in \mathcal{F}(S)$.

Proof. The necessity of $R \in \mathcal{F}(S)$ follows from Lemma 4.1 and for sufficiency we prove only that $\mathcal{F}(T(+)) \cap\{T=R\} \subset \mathcal{F}(S)$ for all stopping times $T$. Since $R$ and $S$ are both $\mathcal{F}(S)$ measurable, $\mathcal{F}(T(+)) \cap\{T=R=S\}=\mathscr{F}(T(+)) \cap\{T=S\} \cap\{R=S\} \epsilon$ $\mathcal{F}(S)$, and it remains to show that $\mathcal{F}(T(+)) \cap\{T=R<S\} \in \mathcal{F}(S)$ as well. But applying Lemma 4.4 (a) twice we have that $T \wedge S$ is $\mathcal{F}(S-)$ measurable and $\mathcal{F}(T(+)) \cap\{T=R<S\}=\mathcal{F}(T(+)) \cap\{T<S\} \cap\{T \wedge S=R\} \subset \mathcal{F}(S)$.

The final result of this section plays a key role in the proof of Theorem 1 .

Lemma 4.6. For any random time $R$

$$
\mathcal{F}\left(R_{-}\right) \cap\left\{X_{R}=X_{R_{-}}\right\}=\mathcal{F}(R) \cap\left\{X_{R}=X_{R_{-}}\right\} \text {. }
$$

Proof. From the definition of $\mathcal{F}(R)$ and Lemma 4.1 (b) it suffices to show that if $S$ is a stopping time, then $\mathfrak{F}(S(+)) \cap\left\{X_{S_{-}}=X_{S}\right\}=\mathcal{F}(S-) \cap\left\{X_{S_{-}}=X_{S}\right\}$. But a result of Blumenthal and Getoor [2] states that if $\mathcal{G}(S)$ is the completion of $\sigma(X(t \wedge S), 0 \leq t)$ in $\mathcal{F}$, then $\mathcal{G}(S)=\mathfrak{F}(S(+))$. In our notation $\mathcal{G}(S) \subset \mathcal{F}^{* *}(S)$, and it is a triviality to verify that $\mathcal{F}^{* *}(S) \cap\left\{X_{S}=X_{S_{-}}\right\}=\mathfrak{F}\left(s_{-}\right) \cap\left\{X_{S}=X_{S_{-}}\right\}$.

5. Statement and proof of Theorem 1. Throughout the remainder of the paper we will use a fixed probability $P=P^{\mu}$, where $\mu$ is a given initial probability on $E_{\Delta}$, and statements true almost surely refer to $P^{\mu}$. We further assume and fix a given coterminal family $\left\{L^{t}, t \geq 0\right\}$ and the resulting terminal time $T$ as defined in $\$ 3$. We emphasize that it is for simplicity of notation that we have assumed the axioms defining $\left\{L^{t}\right\}$ hold on all of $\Omega$. 
Definition 5.1. $A^{r}=\left\{x \in E_{\Delta}-E_{b}: P^{x}(T>0)=0\right\}, A^{i}=\left\{x \in E_{\Delta}-E_{b}\right.$ : $\left.P^{x}(T>0)=1\right\}$. Thus $A^{r}$ is the set of regular points for $T$ and $A^{i}$ the set of irregular points.

Two useful preliminary results are contained in

Lemma 5.1. Let $\left\{S_{a}, a \in I\right\}$ be a countable family of stopping times. Then

$$
P\left[0 \leq L^{t}<t, L^{t}=S_{\alpha} \text { for some } a \in I, X_{L^{t}} \in A^{r}\right]=0 .
$$

As a consequence

$$
P\left[0<L^{t}<t, X_{L^{t}} \neq X_{L^{t}-}, X_{L^{t}} \in A^{r}\right]=0 .
$$

Proof. Equation (5.1) follows from the strong Markov property and the fact that $L^{t}<t$ implies $T \circ \theta_{L t}>0$. For (5.2) let $R_{m n}$ denote the $n$th jump of magnitude exceeding $1 / m$. Then the set in question is $\left\{0<L^{t}<t, L^{t}=R_{m n}\right.$ for some $m$ and $\left.n, X_{L t} \in A^{r}\right\}$ which has measure zero by (5.1).

The conditional distributions which we shall need are given next. Note that they are defined independently of the initial distribution $\mu$.

Definition 5.2. Let $f \in \hat{\mathcal{E}}$ and $s>0$. Then for $x \in A^{i}$ let

$$
D(x, s, f)=E^{x}\left[f\left(X_{s}\right) \mid T>s\right]
$$

with the convention throughout that $0 / 0=0$. For $x \in A^{r}$ and $u>0$ let

$$
D_{n}(x, u, s, f)=E^{x}\left[f\left(X_{s}\right) \mid T \circ \theta_{u}>s-u, \rho\left(x, X_{u}\right)<1 / n\right],
$$

recalling that $\rho$ is the metric on $E_{\Delta}$. Restricting $u$ to rational values let

$$
\bar{D}_{n}(x, s, f)=\varlimsup_{u \rightarrow 0} D_{n}(x, u, s, f), \quad \underline{D}_{n}(x, s, f)=\underline{\lim }_{u \rightarrow 0} D_{n}(x, u, s, f)
$$

and

$$
\bar{D}(x, s, f)=\overline{\lim }_{n \rightarrow \infty} \bar{D}_{n}(x, s, f), \quad \underline{D}(x, s, f)={\underset{\lim }{n \rightarrow \infty}}_{D_{n}}(x, s, f) .
$$

If $\bar{D}(x, s, f)=\underline{D}(x, s, f)$, we call the common value $D(x, s, f)$.

Definition 5.3. For $f \in \hat{\mathcal{E}}$ let $W_{D}(f)=\{(x, s): D(x, s, f)$ exists $\}$.

Note that by definition $A^{i} \times(0, \infty) \subset \mathbb{W}_{D}(f)$ for all $f$. Also, it is easy to show that since the process is right continuous, the functions $\bar{D}(x, s, f)$ and $\underline{D}(x, s, f)$ are universally measurable in the product space $E_{\Delta} \times(0, \infty)$, and thus so is the set $W_{D}(f)$.

Before stating Theorem 1 we remark once again that the property of nearly quasi-left-continuity is not used in this section. If one simply deletes all mention of $E_{b}$ in Definition 5.1, Theorem 1 will be valid as stated, assuming only the strong Markov property.

Theorem 1. Let $f \in \hat{\mathcal{E}}$ and $t>0$. Then a.s. on $\left\{0 \leq L^{t}<t\right\}$ 
(i) $\left(X_{L t^{\prime}} t-L^{t}\right) \in W_{D}(f)$,

(ii) $E\left[f\left(X_{t}\right) \mid \mathcal{F}\left(L^{t}\right)\right]=D\left(X_{L^{t}}, t-L^{t}, f\right)$.

Proof. It suffices to prove the assertion with sup $|f| \leq 1$, and by virtue of a remark in $\$ 2$ we need consider only $X_{L t} \in A^{i} \cup A^{r}$.

Case 1. $X_{L t} \in A^{i}$. Part (i) needs no proof. To prove (ii) it suffices to verify the assertion for $X_{L^{t}} \in F$, where $F$ is a compact subset of $\left\{x: P^{x}(T \geq \delta) \geq 1 / 2\right\}$ and $\delta>0$. To do this define stopping times $S_{n}, n \geq 0$, recursively by

$$
\begin{aligned}
S_{0} & =0, \\
S_{n+1} & =\left\{\begin{array}{l}
\inf \left\{S_{n}<u<t: X_{u} \in F, T \circ \theta_{u-}=0\right\},(6) \\
t \text { if }\{\}=\varnothing .
\end{array}\right.
\end{aligned}
$$

It is easy to show that the $S_{n}$ are measurable. On $\left\{S_{n}<t\right\}, X_{S_{n}} \in A^{i}$, and so $S_{n}<S_{n+1}$ a.s. Moreover $P\left(S_{n+1}-S_{n}<\delta, S_{n}<t\right) \leq 1 / 2 P\left(S_{n}<t\right)$, and it follows easily that a.s. $S_{n}=t$ for all large $n$. Finally, a.s. on $\left\{0 \leq L^{t}<t, X_{L t} \in F\right\}$ there exists $n>0$ such that $L^{t}=S_{n}<S_{n+1}=t$.

Now by Lemma 4.5 for $\Lambda \in \mathscr{F}\left(L^{t}\right)$ there exists a $\Lambda_{n} \in \mathcal{F}\left(S_{n}(+)\right)$ such that $\left\{X_{L^{t}} \in F\right\} \cap \Lambda \cap\left\{L^{t}=S_{n}\right\}=\Lambda_{n} \cap\left\{L^{t}=S_{n}\right\}$. Hence

$$
\begin{aligned}
& E\left[f\left(X_{t}\right) ; \Lambda, L^{t}<t, X_{L^{t}} \in F\right]=\sum_{n=0}^{\infty} E\left[f\left(X_{t}\right) ; \Lambda_{n}, 0 \leq S_{n}<t, T \circ \theta_{S_{n}}>t-S_{n}\right] \\
& =\sum_{n=0}^{\infty} E\left[E\left[f\left(X_{t}\right) ; T \circ \theta_{S_{n}}>t-S_{n} \mid \mathcal{F}\left(S_{n}(+)\right)\right] ; \Lambda_{n}, 0 \leq S_{n}<t\right] \\
& =\sum_{n=0}^{\infty} E\left[P\left(T \circ \theta_{S_{n}}>t-S_{n} \mid \mathcal{F}\left(S_{n}(+)\right)\right) \cdot D\left(X_{S_{n}, t}-S_{n}, f\right) ; \Lambda_{n}, 0 \leq S_{n}<t\right] \\
& =\sum_{n=0}^{\infty} E\left[D\left(X_{S_{n}, t}-S_{n^{\prime}}, f\right) ; \Lambda, 0 \leq S_{n}=L^{t}<t, X_{L^{t}} \in F\right] \\
& =E\left[D\left(X_{L^{t}}, t-L^{t}, f\right) ; \Lambda, L^{t}<t, X_{L^{t}} \in F\right] .
\end{aligned}
$$

Case 2. $X_{L t} \in A^{r}$.

Remark. Since the proof in this case is rather involved, we have divided it into six steps with the first step reducing the problem to showing that a particular inequality (equation (5.3)) suffices to prove the theorem. In the second step we introduce some notation required to define the families of stopping times $\left\{S_{a}^{m}\right\}$

(6) The notation $T \circ \theta_{u-}$ denotes $\lim _{\nu \uparrow u} T \circ \theta_{v}$. 
given in the third step. Each family is so constructed that a.s. on $\Gamma=$ $\left\{L^{t}<t,\left(X_{L t^{\prime}} L^{t}\right) \in K\right\}, K$ a compact subset of $E_{\Delta} \times[0, t)$, for each $m$ there is an a such that

$$
0<L^{t}-S_{a}^{m}<S_{a+1}^{m}-S_{a}^{m}<\delta_{m}
$$

where $\delta_{m} \downarrow 0$. Moreover when the strong Markov property is applied to $S_{a}^{m}$ on $\left\{S_{a}^{m}<L^{t}<S_{a+1}^{m}\right\}$, the resulting expression can be approximated first by $\bar{D}_{n}\left(X_{s_{a}^{m}}, t-S_{a}^{m}, f\right)$ for some $n \geq m$ and then by $\bar{D}\left(X_{L t^{\prime}} t-L^{t}, f\right)$, and this double approximation is at the heart of the proof. In Step 4 we patch together the $S_{a}^{m}$ to define random times $R^{m}$ which permit us to obtain $(5.3)$ in the next step, subject to a technicality which is removed in the last step.

(1) Reduction of the proof to (5.3). Let $\epsilon>0$ and suppose $-1=a_{0}<$ $a_{1}<\ldots<a_{N}$ with $1<a_{N}$ and $a_{l+1}-a_{l}<\epsilon$. For $1 \leq l \leq N$ let

$$
B_{l}=\left\{(x, s): a_{l-1} \leq \bar{D}(x, t-s, f)<a_{l}, x \in A^{r}\right\} .
$$

Since $B_{l}$ is universally measurable, there exist compact sets $K_{l} \subset B_{l}$ such that

$$
P\left[L^{t}<t,\left(X_{L}, L^{t}\right) \in B_{l}-K_{l}\right]<\epsilon \cdot 2^{-l} .
$$

Now suppose we could show that

$$
\left|E\left[f\left(x_{t}\right) ; \Lambda, \Gamma_{l}\right]-E\left[\bar{D}\left(X_{L^{t}}, t-L^{t}, f\right) ; \Lambda, \Gamma_{l}\right]\right| \leq \epsilon P\left(\Lambda \cap \Gamma_{l}\right)
$$

where $\Lambda \in \mathcal{F}\left(L^{t}\right)$ and $\Gamma_{l}=\left\{L^{t}<t,\left(X_{L t^{\prime}} L^{t}\right) \in K_{l}\right\}$. Then after summing on $l$ and using the fact that $\epsilon$ is arbitrary we would have

$$
\cdot E\left[f\left(X_{t}\right) ; L^{t}<t, \Lambda\right]=E\left[\bar{D}\left(X_{L^{t}}, t-L^{t}, f\right) ; L^{t}<t, \Lambda\right] .
$$

Moreover, if the argument were only notationally different for $\underline{D}$, both parts of the theorem would follow.

Thus, dropping the subscripts, it suffices to show

$$
\left|E\left[f\left(x_{t}\right) ; \Lambda, \Gamma\right]-E\left[\bar{D}\left(x_{L} t^{, t}-L^{t}, f\right) ; \Lambda, \Gamma\right]\right| \leq \epsilon P(\Lambda \cap \Gamma),
$$

where $\Lambda \in \mathcal{F}\left(L^{t}\right)$,

$$
\Gamma=\left\{L^{t}<t,\left(X_{L}{ }^{t}, L^{t}\right) \in K\right\}
$$

and $K$ is a compact set of the product space $E_{\Delta} \times[0, t)$ such that for $(x, s)$ and $(y, r)$ in $K$

$$
|\bar{D}(x, t-s, f)-\bar{D}(y, t-r, f)|<\epsilon .
$$

At the conclusion of the proof the reader can see that the argument is the same for $\underline{D}$. 
$\langle 2\rangle$ Preliminary definitions. For any $\delta>0$ it is possible to find an increasing sequence of integers $\left\{N_{m}, m<N_{m}\right\}$ and a compact set $\hat{K} \subset K$ such that for all $(x, s) \in \hat{K}$ and $m \geq 1$ there exists an $n$ with $m \leq n<N_{m}$ and

$$
\left|\bar{D}(x, t-s, f)-\bar{D}_{n}(x, t-s, f)\right|<1 / m,
$$

and such that $P\left[L^{t}<t,\left(X_{L t^{\prime}} L^{t}\right) \in K-\hat{K}\right]<\delta$. Thus we may assume that $K$ itself possesses such a sequence, and we define for each $m$ and $(x, s) \in K$

$$
\bar{n}=\bar{n}_{m}(x, s)=\min \left\{n: m \leq n<N_{m}:(5.6) \text { holds }\right\} .
$$

By virtue of Lemma 5.1 we have continuity at $L^{t}$ a.s. on $\Gamma$ and can find a sequence of $\delta_{m} \downarrow 0$ such that

$$
\begin{aligned}
& P\left[\Gamma, \text { for some rational } r_{1} \text { and } r_{2} \text { in }\left(L^{t}-\delta_{m}, L^{t}+\delta_{m}\right),\right. \\
& \left.\qquad \rho\left(X_{r_{1}}, X_{r_{2}}\right) \geq 1 / N_{m}\right]<2^{-m} .
\end{aligned}
$$

By $R^{\#}$ we will denote an enumeration of the rationals in $(0, t)$. Then for $s<v \leq t$ and $(x, s) \in K$ define

$$
\begin{aligned}
& \bar{u}=\bar{u}_{m}(x, s, v)=\text { first } u \in R^{\#} \text { with } u<(v-s) \wedge \delta_{m} \text { and } \\
&\left|\bar{D}_{\bar{n}}(x, t-s, f)-D_{\bar{n}}(x, u, t-s, f)\right|<1 / m .
\end{aligned}
$$

〈3〉 Construction of $\left\{S_{a}^{m}\right\}$. For $m=0$ let $S_{0}^{0}=0$ and $S_{1}^{0}=t$. For $m=1$ we define an increasing sequence of stopping times indexed by the countable ordinals, where we call an ordinal $\alpha$ even if $\alpha=\beta+2 n$ with $\beta$ zero or a limit ordinal and $n$ a finite ordinal. For all even $\alpha$

$$
S_{a}^{1}=\inf \left\{u \geq \sup _{\beta<a} S_{\beta}^{1}:\left(X_{u}, u\right) \in K\right\},
$$

interpreting $\sup _{\beta<0} S_{\beta}^{1}$ as zero. Here and subsequently we assign the value $t$ if the defining set is empty. Next define

$$
S_{a+1}^{1}=\left\{\begin{array}{l}
S_{a}^{1}+\bar{u}_{1}\left(X_{S_{a}^{1}}, S_{a}^{1}, t\right) \text { if } S_{a}^{1}<t \\
t \text { if } S_{a}^{1}=t .
\end{array}\right.
$$

All of the $S_{\alpha}^{1}$ are stopping times. (7) If $\alpha$ is even and $0<S_{\alpha}^{1}<t$, then since $X_{s_{\alpha}^{1}} \in A^{r}, T \circ \theta_{s_{a}^{1}}=0$. From the definition $S_{a}^{1}<S_{a+2}^{1}$ which implies that

(1) There exists a countable ordinal $\beta_{1}$ such that $S_{\beta_{1}}^{1}=t$ a.s. In addition

(7) For a even, $S_{\alpha}^{1}$ is of the form $\min \{u:(u, \omega) \in \mathbf{\Lambda}\}$, where $\Lambda=\{(u, \omega): u \geq R$, $\left.\left(X_{u}, u\right) \in K\right\}$ with $R$ a stopping time. $S_{a}^{1}$ is then a stopping time by [5, IV, T52] and $S_{a+1}^{1}$ a stopping time by definition. 
we have

(2) If $a$ is even, $S_{a+1}^{1} \in \mathcal{F}\left(S_{a}^{1}(+)\right)$.

(3) If $\alpha$ is even and $S_{\alpha}^{1}<t$, then $S_{a+1}^{1}<t,\left(X_{S_{\alpha}^{1}}, S_{a}^{1}\right) \in K$ and $0<S_{a+1}^{1}-$ $S_{a}^{1}<\delta_{1}$.

(4) If $u \in \bigcup_{\text {even }} a<\beta_{1}\left[\bigvee_{\beta<a} S_{\beta}^{1}, S_{a}^{1}\right)$, then $\left(X_{u}, u\right) \notin K$.

(5) If $\alpha$ is even and $S_{\alpha}^{1}<t$, there is an even $\gamma$ such that

$$
S_{\gamma}^{0} \leq S_{a}^{1}<S_{a+1}^{1}<S_{\gamma+1}^{0}
$$

(6) If $\gamma$ is even and $S_{\gamma}^{0}<t$, there is an even a such that (5.10) holds.

Properties (5) and (6) are trivial of course, but we include them to simplify the induction statements below. Note that by Lemma 5.1 and (4) above, a.s. on $\Gamma$ there is some even $a$ with $S_{\alpha}^{1}<L^{t}<S_{a+1}^{1}<t$.

Next assume a family $\left\{S_{a}^{m}\right\}$ of increasing stopping times has been defined which satisfies (1) through (6) with $m$ replacing 1 as a superscript and $(m-1)$ replacing 0 as a superscript in (5) and (6). Let $S_{0}^{m+1}=S_{0}^{m}$ and

$$
S_{1}^{m+1}=\left\{\begin{array}{l}
S_{0}^{m+1}+\bar{u}_{m+1}\left(X_{S_{0}^{m+1}}, S_{0}^{m+1}, U_{0}^{m+1}\right) \text { if } S_{0}^{m+1}<t, \\
t \quad \text { if } S_{0}^{m+1}=t,
\end{array}\right.
$$

where $U_{0}^{m+1}=S_{1}^{m}$. By (2) above $S_{1}^{m+1} \in \mathcal{F}\left(S_{0}^{m+1}(+)\right)$. Again for a even let

$$
S_{a}^{m+1}=\inf \left\{u \geq \sup _{\beta<a} S_{\beta}^{m+1}:\left(X_{u}, u\right) \in K\right\}
$$

By virtue of (4)

$$
U_{a}^{m+1}=\left\{\begin{array}{l}
S_{\gamma+1}^{m} \text { if } S_{\gamma}^{m} \leq S_{a}^{m+1}<s_{\gamma+1}^{m}, \gamma \text { even } \\
t \text { if no such } \gamma \text { exists }
\end{array}\right.
$$

is well defined, and by (2), $U_{a}^{m+1} \in \mathcal{F}\left(S_{a}^{m+1}(+)\right)$. Then let

$$
S_{a+1}^{m+1}=\left\{\begin{array}{l}
S_{a}^{m+1}+\bar{u}_{m+1}\left(X_{S_{a}^{m+1}}, S_{a}^{m+1}, U_{a}^{m+1}\right) \text { if } S_{a}^{m+1}<t, \\
t \quad \text { if } S_{a}^{m+1}=t,
\end{array}\right.
$$

so that if $S_{\alpha}^{m+1}<t,\left(X_{S_{\alpha}^{m+1}}, S_{\alpha}^{m+1}\right) \in K$ and $S_{a+1}^{m+1}<U_{a}^{m+1}$.

As with $m=1$ the $\left\{S_{a}^{m+1}\right\}$ are increasing stopping times, and (1) through (4) hold at the $(m+1)$ th level. Properties (5) and (6) follow by induction. For example, if (5) is true for all $\beta<\alpha, \alpha$ is even, then either $\sup _{\beta<\alpha} s_{\beta}^{m+1} \epsilon$ $\left[s_{\gamma}^{m}, s_{\gamma+1}^{m}\right)$ for some even $\gamma$, and (5) is immediate for $\alpha$, or else $\sup _{\beta<\alpha} s_{\beta}^{m+1}$ equals $S_{\gamma+1}^{m}$ or $\sup _{\gamma^{\prime}<\gamma} S_{\gamma}^{m}$, for some even $\gamma$ and then $S_{a}^{m+1}=S_{\gamma+2}^{m}$. Again, 
a.s. on $\Gamma$ there is an even $\alpha$ with $S_{\alpha}^{m}<L^{t}<S_{\alpha+1}^{m}<t$.

〈4) Random times approximating $L^{t}$. We may now choose a countable ordinal $\beta_{0}$ such that $S_{\beta_{0}}^{m}=t$ a.s. for all $m$. Also, a.s. on $\Gamma$ for each $m>0$ there exist even $\alpha$ and $\gamma<\beta_{0}$ such that

$$
S_{\gamma}^{m} \leq S_{a}^{m+1}<L^{t}<S_{a+1}^{m+1}<S_{\gamma+1}^{m}<t
$$

By Lemma 4.5,

$$
\mathcal{F}\left(S_{\alpha}^{m}(+)\right) \cap\left\{S_{a}^{m}<L^{t}\right\} \subset \mathcal{F}\left(L^{t}-\right)
$$

and hence

$$
R_{m}= \begin{cases}S_{a}^{m} & \alpha \text { is even and } S_{a}^{m}<L^{t}<S_{a+2}^{m} \\ t & \text { otherwise, }\end{cases}
$$

is well defined and $\mathcal{F}\left(L^{t}-\right)$ measurable. Note that $R_{m} \leq R_{m+1}$ and $0<L^{t}-$ $R_{m}<\delta_{m}$ if $R_{m}<t$. Hence up to sets of measure zero

$$
\Gamma \subset\left\{R_{m}<L^{t}=\lim _{m^{\prime} \rightarrow \infty} R_{m^{\prime}}, \text { all } m\right\}=\left\{R_{m}<t \text {, all } m\right\}
$$

Note also that $R_{m}$ is honest. (8)

(5) Proof of (5.3). Let $\Lambda \in \mathcal{F}\left(R_{m_{0}}(+)\right), m>m_{0}$ and fix $\alpha<\beta_{0}$. Then since $\mathcal{F}\left(R_{m_{0}}(+)\right) \subset \mathcal{F}\left(R_{m}(+)\right)$ by Lemma 4.2 , by Lemma 4.5 there exists a $\Lambda_{a} \epsilon$ $\mathcal{F}\left(S_{a}^{m}(+)\right)$ with $\Lambda \cap\left\{R_{m}=S_{a}^{m}\right\}=\Lambda_{a} \cap\left\{R_{m}=S_{a}^{m}\right\}$. If

$$
\Gamma_{a}^{m}=\left\{S_{a}^{m}<L^{t}<S_{a+1}^{m}, \rho\left(X_{s_{a}^{m}}, X_{s_{a+1}^{m}}\right)<1 / \bar{n}\right\},
$$

where $\bar{n}=\bar{n}_{m}\left(X_{s_{a}^{m}}, S_{a}^{m}\right)$ is defined in (5.7), then $\Lambda \cap \Gamma_{a}^{m}=\Lambda_{a} \cap \Gamma_{a}^{m}$. An application of the strong Markov property similar to that in Case 1 gives

$$
\begin{aligned}
& E\left[f\left(X_{t}\right) ; \Lambda, \Gamma_{a}^{m}\right] \\
& \quad=E\left[E^{X\left(S_{a}^{m}\right)}\left[f\left(X_{t-s_{a}^{m}}\left(\omega^{\prime}\right)\right) ; t-S_{a}^{m}<T \circ \theta_{\bar{u}} \omega^{\prime}, \rho\left(X_{s_{a}^{m},} X_{\bar{u}}\left(\omega^{\prime}\right)\right)<1 / \bar{n}\right] ;\right. \\
& \quad=E\left[D_{\bar{n}}\left(X_{s_{a}^{m}}, \bar{u}_{a}, t-S_{a}^{m}<t\right]\right.
\end{aligned}
$$

where $\bar{u}=\bar{u}_{m}\left(X_{s_{a}^{m}}, S_{a}^{m}, U_{a}^{m}\right)$ and $U_{a}^{m}$ is defined in equation (5.11). But then by (5.6) and (5.7)

(8) For $u<v \leq t,\left\{R_{m}<u\right\} \cap\left\{R_{m}<v\right\}=\bigcup_{\text {even } a<\beta_{0}}\left\{S_{a}^{m}<u, S_{a+2}^{m} \geq v\right\} \cap\left\{R_{m}<v\right\}$. 
$(5.15)$

$$
\left|E\left[f\left(X_{t}\right) ; \Lambda, \Gamma_{a}^{m}\right]-E\left[\bar{D}\left(X_{s_{a}^{m}}, t-s_{a}^{m}, f\right) ; \Lambda, \Gamma_{a}^{m}\right]\right| \leq(2 / m) P\left(\Lambda \cap \Gamma_{a}^{m}\right) .
$$

Suppose now that we could show that

$$
P\left(\Gamma \Delta \Gamma^{m}\right) \rightarrow 0
$$

where $\Gamma^{m}=\bigcup_{a<\beta_{0}} \Gamma_{a}^{m}$. Then adding (5.15) over $a<\beta_{0}$ and using (5.5) we would have (5.3) in the limit for $\Lambda \in \mathcal{F}\left(R_{m_{0}}(+)\right)$. By virtue of Lemmas 4.4 and 4.6

$$
\begin{aligned}
\mathcal{F}\left(L^{t}\right) \cap\left\{R_{m}<L^{t},\right. \text { all } & m\} \cap\left\{X_{L^{t}}=X_{L^{t}-}\right\} \\
= & \bigvee_{m} \mathfrak{F}\left(R_{m}(+)\right) \cap\left\{R_{m}<L^{t} \text {, all } m\right\} \cap\left\{X_{L^{t}}=X_{L^{t}-}\right\},
\end{aligned}
$$

and thus, by (5.13), $\mathcal{F}\left(L^{t}\right) \cap \Gamma=\bigvee_{m} \mathcal{F}\left(R_{m}(+)\right) \cap \Gamma$. Finally, since $\mathcal{F}\left(R_{m}(+)\right)$ is increasing (Lemma 4.2), for $\Lambda \in \mathcal{F}\left(L^{t}\right)$ there exist $\Lambda_{m} \in \mathcal{F}\left(R_{m}(+)\right)$ with $P\left[\Gamma \cap\left(\Lambda \Delta \Lambda_{m}\right)\right] \rightarrow 0$. Thus (5.3) must hold for all $\Lambda \in \mathcal{F}\left(L^{t}\right)$.

$\langle 6\rangle$ Verification of (5.16). If $\omega \notin \Gamma$, then $\left(X_{L t}, L^{t}\right) \notin K$ so that $\left(X_{L^{t}}, L^{t}\right)$ is not a limit point of $\left(X_{R_{m}}, R_{m}\right)$. Hence $\omega \notin \Gamma^{m}$ for all large $m$. On the other hand if $\omega \in \Gamma$, then a.s. for each $m$ and some even $\alpha<\beta_{0}, R^{m}=S_{a}^{m}<L^{t}<$ $s_{a+1}^{m}<t$. Because of (5.8), for large $m$

$$
\rho\left(X_{s_{a}^{m}}, X_{s_{a+1}^{m}}\right)<1 / N_{m}<\left(\bar{n}_{m}\right)^{-1}
$$

and hence $\omega \in \Gamma^{m}$ for sufficiently large $m$. This completes the verification of (5.16), and since the argument is similar for $\underline{D}$ in lieu of $\bar{D}$, the proof of the theorem is complete.

6. Statement and Proof of Theorem 2. In this section we deal with conditioning by $\mathcal{F}\left(L^{t_{-}}\right)$and thus need to assume the property of nearly quasi-left continuity as defined in $\$ 2$. The proof of Theorem 2 is basically a somewhat simplified version of the proof of Theorem 1 , but the loss of information at $L^{t}$ causes some additional complications. Before going into details, we define the conditional probabilities $Q^{b}$.

Definition 6.1. Suppose $b$ and $f$ are in $\hat{\varepsilon}$ and $s>0$. Retaining the convention $0 / 0=0$, define for $x \in E_{b}$

$$
Q^{h}(x, s, f)=E^{x}\left[b\left(X_{0}\right) f\left(X_{s}\right) \mid T>s\right]=\frac{\int \nu(x, d y) b(y) E^{y}\left[f\left(X_{s}\right) ; T>s\right]}{\int \nu(x, d y) P^{y}(T>s)} .
$$

For $x \in E_{r}$ (recall that $E_{r}=E_{\Delta}-E_{b}$ ) and $0<u<s$, let

$$
Q^{b}(x, u, s, f)=E^{x}\left[b\left(X_{u}\right) f\left(X_{s}\right) \mid T \leq u, T \circ \theta_{u}>s-u\right] .
$$

With $u$ restricted to the rationals, define 


$$
\bar{Q}^{h}(x, s, f)=\varlimsup_{u \rightarrow 0} Q^{h}(x, u, s, f)
$$

and

$$
\underline{Q}^{b}(x, s, f)=\frac{\lim }{u \rightarrow 0} Q^{b}(x, u, s, f)
$$

If $(x, s)$ is in

$$
W_{Q}(b, f)=\left\{(x, s) \in E_{\Delta} \times(0, \infty): \bar{Q}^{b}(x, s, f)=\underline{Q}^{b}(x, s, f)\right\},
$$

let $Q^{b}(x, s, f)$ denote the common value. If $b=1$, we suppress the superscript and write $Q(x, s, f)$.

The difficulty we encounter now is that $Q^{b}\left(X_{L}, t_{-} L^{t}, f\right)$ is not always the correct conditional probability on $\left\{0<L^{t}<t, X_{L^{t}-} \in A^{i} \cup E_{b}\right\}$. To illustrate the case $X_{L_{-}} \in A^{i}$, let the process be uniform motion to the right on $[-1,0)$ followed by an exponentially distributed hold at $\{0\}$ and a subsequent jump to the absorbing point $\{1\}$. Then if $L^{t}=\sup \left\{u \leq t: X_{u} \neq X_{u-}\right.$ or $\left.X_{u} \in[-1,0)\right\}$, it follows that $X_{L^{t}-}=0$ on $\left\{0<L^{t}<t\right\}$ but $X_{L^{t}}$ may be 0 or 1 . If $X_{L^{t}}=0$, definition 6.1 gives the correct distribution $h(0) \cdot f(0)$, but if $X_{L t}=1$ we should use $b(0) \cdot f(1)$. In other words, for $x=X_{L^{t}-} \in A^{i}$, two different conditional distributions may be associated with $x$. We shall show below that this behavior is the most general possible; i.e., on a set $\Psi_{t}$ in $\mathcal{F}\left(L^{t}-\right)$ and contained in $\left\{0<L^{t}<t, X_{L^{t}-} \in A^{i}\right\}$ one family of conditional distributions is appropriate, while on $\left\{0<L^{t}<t, X_{L^{t}-} \epsilon A^{i}\right\}-\Psi_{t}$ the family $Q^{b}$ is correct. (9)

On $\left\{0<L^{t}<t, X_{L^{t}-} \in E_{b}\right\}$ the conditional distributions $Q^{b}$ again need not be correct. For example if $F$ is a compact subset of $E_{r}$ and $L^{t}=$ $\sup \left\{u \leq t: X_{u} \in F\right\}$, then the correct conditional distributions for $x \in E_{b}$ are defined as in Definition 6.1 but with the integral taken only over $F$. With such changes in $Q^{b}(x, s, f)$ Theorem 2 will hold for special cases on all of $\left\{0<L^{t}<t_{,} X_{L^{t}-} \in E_{b}\right\}$, but our concern here will be with defining a subset $\Phi_{t}$ of $\left\{0<L^{t}<t, X_{L^{t}-} \in E_{b}\right\}$ for which Definition 6.1 gives the correct distributions.

To define $\Psi_{t}$ we need two additional concepts about random times; the first is a standard one.

Definition 6.2. A stopping time $S$ is said to be previsible if there exist stopping times $\tau_{n}<S=\lim \tau_{n}$ a.s. on $\{S>0\}$ for all $n .(10)$

(9) We are indebted to J. Walsh for the above example and for pointing out several errors in the original version of Theorem 2.

$(10)$ Note that nearly quasi-left continuity can be formulated as $p^{\mu}\left(X_{S} \epsilon \cdot \mid \xi(S-)\right)=$ $\nu\left(X_{S_{-}}, \cdot\right)$ a.s. on $\{0<S<\infty\}$ for previsible stopping times $S$. 
Definition 6.3. Let $R$ be a random time and $\Lambda \subset\{0<R<\infty\} . R$ is said to be accessible on $\Lambda$ if for each initial measure $\gamma$ there exist previsible stopping times $S_{m}, m \geq 1$, such that, a.s. $P^{\gamma}, \Lambda \subset \bigcup_{m}\left\{R=S_{m}\right\}$.

Recall that we are assuming a fixed probability $P=P^{\mu}$. Then

Definition 6.4. Let $\Psi_{t}$ be the subset of $\left\{0<L^{t}<t, X_{L^{t}-} \in A^{i}\right\}$ such that $L^{t}$ is accessible on $\Psi_{t}$ and $P\left(\Psi_{t}\right)$ is maximal.

The existence of such a $\Psi_{t}$ is easy to verify while the implied uniqueness is modulo $P$-null sets.

Lemma 6.1. $\Psi_{t} \in \mathcal{F}\left(L^{t_{-}}\right)$and $X_{L^{t}}=X_{L^{t}-}$ a.s. on $\Psi_{t} \cdot{ }^{(11)}$

Proof. By definition $\Psi_{t}$ will be of the form $\bigcup_{m}\left\{L^{t}=S_{m}, 0<L^{t}<t, X_{L^{t}-} \in A^{i}\right\}$ for a countable collection of previsible stopping times $S_{m}$. Then $\Psi_{t} \in \mathcal{F}\left(L^{t}-\right)$ by Lemma $4.5(\mathrm{a})$, and, since $X_{L^{t}-} \not E_{b}, X_{L^{t}}=X_{L^{t}-}$ a.s. on $\Psi_{t}$ by nearly quasi-left continuity.

Note that by the maximality of $\Psi_{t}$, up to $P$-null sets $L^{t}$ is not equal to a previsible stopping time on $\left\{0<L^{t}<t, X_{L^{t}-} \in A^{i}\right\}-\Psi_{t}$. We formalize that property in

Definition 6.5. A random time $R$ is totally inaccessible on $\Lambda$ relative to $P^{\gamma}$ if $P^{\gamma}(R=S, \Lambda)=0$ for all previsible $S . R$ is totally inaccessible on $\Lambda$ if it is totally inaccessible on $\Lambda$ relative to $P^{\gamma}$ for all $\gamma$.

As an illustration of these definitions we have

Lemma 6.2. $L^{t}$ is totally inaccessible on $\left\{0<L^{t}<t, X_{L^{t}-} \in A^{r}\right\}$ and accessible on $\left\{0<L^{t}<t_{0} X_{L^{t}-} \in E_{b}\right\}$.

Proof. The first assertion follows from the nearly quasi-left continuity and Lemma 5.1. For the second assertion it suffices to show that $L^{t}$ is accessible on $\left\{0<L^{t}<t, X_{L^{t}-} \in \bigcup_{n} F_{n}\right\}$, where the $F_{n}$ are compact subsets of $E_{b}$ and $P\left(0<L^{t}<t, X_{L^{t}-} \in E_{b}-\bigcup_{n} F_{n}\right)=0$. By Lemma 6.5 below we can choose such $F_{n}$ so that $L^{t}$ equals one of the countable family of previsible times inf $\left\{s>r, X_{s-} \in F_{n}\right\}, r$ rational, and this completes the proof.

The key to the proof of Theorem 2 on the totally inaccessible set $\left\{0<L^{t}<t, X_{L^{t}-} \in E_{r}\right\}-\Psi_{t}$ relative to $P$ is given in

(11) Under reasonable assumptions on the process, it is also true that $X_{L^{t}} \not X_{L^{t}-}$ on $\left\{0<L^{t}<t, X_{L^{t}-} \epsilon A^{i}\right\}-\Psi_{t}$, so that $\Psi_{t}$ would equal $\left\{0<L^{t}<t, X_{L^{t}-}=X_{L^{t}} \epsilon A^{i}\right\}$, which is independent of the measure $P$. See P. A. Meyer and J. L. Walsh: Quelques applications des resolvantes de Ray, Invent. Math. 14 (1971), 143-166. 
Lemma 6.3. Let $K$ be a compact subset of $E_{r} \times(0, t)$. Then a.s. on $\left\{0<L^{t}<t,\left(X_{L^{t}-}, L^{t}\right) \in K\right\}-\Psi_{t}$

$$
L^{t}=\sup \left\{u<L^{t}:\left(X_{u}, u\right) \in K\right\} .
$$

Proof. If not, then for some $u_{0}$ the set $\Lambda=\left\{0<L^{t}<t, X_{L^{t}-} \in E_{r}\right.$, $\left(X_{u}, u\right) \not k K$ for all $\left.u \in\left(u_{0}, L^{t}\right)\right\}-\Psi_{t}$ has positive measure. If $\rho_{1}$ is the metric on $E_{\Delta} \times[0, \infty)$ given by $\rho_{1}((x, u),(y, v))=\rho(x, y)+|u-v|$ and $\tau_{n}=$ inf $\left\{u>u_{0}: \rho_{1}\left(\left(X_{u}, u\right), K\right)<1 / n\right\}$, we have $\tau_{n}<L^{t}=\lim _{n} \tau_{n}$ a.s. on $\Lambda$. This contradicts the total inaccessibility relative to $P$, and the lemma is proved.

Our next task before proving Theorem 2 is to define the subset $\Phi_{t}$ of $\left\{0<L^{t}<t, X_{L^{t}-} \in E_{b}\right\}$. Ideally we would like to give a definition which is independent of the measure P: e.g., $\Phi_{t}=\left\{0<L^{t}<t, X_{L^{t}-} \in E_{b^{\prime}} L^{t} \circ k_{L^{t}}=L^{t}\right\}$. Unfortunately in order to show $\Phi_{t} \in \mathfrak{F}\left(L^{t}-\right)$ and the crucial property (6.1) below, it seems to be necessary to make a technical assumption such as $L^{t} \circ k_{S} \epsilon$ $\mathcal{F}\left(S_{-}\right)$for all previsible stopping times $S$. To avoid such an assumption we use instead

Definition 6.6. Let $\Phi_{t}$ denote the subset of $\left\{0<L^{t}<t, X_{L^{t}-} \in E_{b}\right\}$ with maximal $P\left(\Phi_{t}\right)$ and satisfying: for any-previsible stopping time $S$ there is a $\Gamma_{S} \in \mathcal{F}\left(S_{-}\right)$contained in $\{0<S<t\}$ such that

$$
\Phi_{t} \cap\left\{L^{t}=S\right\}=\Gamma_{S} \cap\left\{T \circ \theta_{S}>t-S\right\}
$$

Intuitively our motivation is to define a set on which $L^{t}$ is independent of $X_{L^{t}}$ and depends only on information up to $L^{t}$ and on $T \circ \theta_{L^{t}}>t-L^{t}$ (i.e. $\left.L^{t} \circ k_{L^{t}}=L^{t}\right)$. Note that for the second example given above $\Phi_{t}$ is empty since $L^{t}<t$ forces $X_{L^{t}} \in F$.

Lemma 6.4. $\Phi_{t} \in \mathcal{F}\left(L^{t}-\right)$.

Proof. As noted earlier $L^{t}$ equals one of a countable number of previsible stopping times on $\left\{0<L^{t}<t_{0} X_{L^{t}-} \in E_{b}\right\}$, and thus it suffices to show $\Phi_{t} \cap$ $\left\{L^{t}=S\right\} \in \mathscr{F}\left(L^{t}-\right)$ for a previsible stopping time $S$. By (6.1), $\Phi_{t} \cap\left\{L^{t}=S\right\}=$ $\Gamma_{S} \cap\left\{0<L^{t}=S<t\right\}$ with $\Gamma_{S} \in \mathcal{F}(S-)$, and by Lemma $4.1(\mathrm{~b})$ this is in $\mathcal{F}\left(L^{t}\right) \cap$ $\left\{0<L^{t}=S<t\right\}$. Lemma $4.5\left(\right.$ a) and previsibility give $\left\{0<L^{t}=S<t\right\} \in \mathcal{F}\left(L^{t}-\right)$, completing the proof.

Definition 6.7. $\Omega_{t}=\Phi_{t} \cup\left(\left\{0<L^{t}<t, X_{L^{t}-} \in E_{r}\right\}-\Psi_{t}\right)$. Note that $\Omega_{t}$ depends on $P$ and that $\Omega_{t} \in \mathcal{F}\left(L^{t}-\right)$.

Before stating Theorem 2 we need one final result.

Lemma 6.5. Let $\delta>0$ and let $F$ be a compact subset of $\left\{x \in E_{b}\right.$ : $\nu(x, B(x, \delta))<1 / 2\}$ where $B(x, \delta)=\left\{y \in E_{\Delta}: \rho(x, y)<\delta\right\}$ (recalling that $\nu$ is the 
brancbing measure). Then a.s. $\left\{u>0, X_{u_{-}} \in F\right\}$ is separated, i.e. has no finite accumulation points.

Proof. It is obvious that only a finite number of $u$ with $\rho\left(X_{u-}, X_{u}\right) \geq \delta$ can occur in finite time. Let

$$
S_{1}=\inf \left\{u>0: X_{u-} \in F, \rho\left(X_{u^{\prime}}, X_{u-}\right)<\delta, X_{s-} \notin F \text { for } 0<s<u\right\}
$$

and

$$
S_{n+1}=S_{n}+S_{1} \circ \theta_{S_{n}},
$$

where $S_{n+1}=\infty$ if $S_{n}=\infty$. (12) If the lemma is false, there must be some $u \geq 0$ such that on a set of positive measure, $S_{n} \circ \theta_{u}<\infty$ for all $n$. Thus we can establish the lemma by proving $P^{x}\left(S_{n}<\infty\right.$ for all $\left.n\right)=0$ for any $x \in E$. This will follow from $P^{x}\left(S_{n+1}<\infty\right) \leq 1 / 2 P^{x}\left(S_{n}<\infty\right)$ which in turn will follow from $P^{x}\left(S_{1}<\infty\right) \leq 1 / 2$. To show this, let $S=\inf \left\{u>0, X_{u-} \in F\right\}$. It is easy to see that $S>0$ a.s. and is previsible. Hence by nearly quasi-left continuity

$$
\begin{aligned}
P^{x}\left(s_{1}<\infty\right) & =P^{x}\left(s<\infty, \rho\left(X_{S_{-}}, X_{S}\right)<\delta\right) \\
& =E^{x}\left(\nu\left(X_{S_{-}}, B\left(X_{S_{-}}, \delta\right)\right), s<\infty\right) \leq 1 / 2 .
\end{aligned}
$$

Theorem 2. Let $t>0, f \in \hat{\mathcal{G}}$ and $b \in \mathcal{C}$. Then almost surely on $\Omega_{t}$

(i) $\left(X_{L^{t}-}, t-L^{t}\right) \in W_{Q}(b, f)$, and

(ii) $E\left[b\left(X_{L^{t}}\right) f\left(X_{t}\right) \mid \mathcal{F}\left(L^{t}-\right)\right]=Q^{b}\left(X_{L^{t}-}, t-L^{t}, f\right)$.

Almost surely on $\Psi_{t},\left(X_{L^{t}-}, t-L^{t}\right) \in W_{D}(f)$ and

$$
E\left[b\left(X_{L^{t}}\right) f\left(X_{t}\right) \mid \mathcal{F}\left(L^{t}-\right)\right]=b\left(X_{L^{t}-}\right) D\left(X_{L^{t}-}, t-L^{t}, f\right) .
$$

Remark. If we weaken the nearly quasi-left continuity by requiring (2.2) to hold a.s. on $\left\{T_{n}<T<\zeta\right.$, all $\left.n\right\}$, the theorem and subsequent results based on it are still valid provided $\Omega_{t}$ and $\Psi_{t}$ are replaced by $\Omega_{t} \cap\left\{L^{t}<\zeta\right\}$ and $\Psi_{t} \cap$ $\left\{L^{t}<\zeta\right\}$ respectively.

Proof of Theorem 2. Since $\mathcal{F}\left(L^{t_{-}}\right) \subset \mathcal{F}\left(L^{t}\right)$ and $X_{L^{t}-} \in A^{i}$ on $\Psi_{t}$ the second assertion is immediate from Theorem 1. For the first part of the theorem we consider the two sets $\Phi_{t}$ and $\left\{0<L^{t}<t, X_{L^{t}-} \in E_{r}\right\}-\Psi_{t}$ separately, and note that it suffices to assume $f \in \mathcal{E}$ and both $f$ and $b$ are bounded in absolute value by 1 .

Case 1. $X_{L^{t}-} \in E_{b}$. In this case (i) is immediate, and it suffices to prove (ii) on $\Phi_{t} \cap\left\{X_{L^{t}-} \in F\right\}$, where $F$ is a compact subset of $\left\{x \in E_{b}: \nu(x, B(x, \delta))<\right.$ $1 / 2\}, \delta>0$. Define stopping times $\left\{S_{n}, n>0\right\}$ by $S_{0}=0$ and

(12) By remarks in $\S 2, X_{S_{n}}, F$ a.s. and since $F$ is compact it follows that $S_{n}<$ $S_{n+1}$ on $\left\{S_{n}<\infty\right\}$. 


$$
S_{n+1}=\left\{\begin{array}{l}
\inf \left\{u: S_{n}<u, t_{,} X_{u-} \in F\right\} \\
t \quad \text { if }\{\}=\varnothing .
\end{array}\right.
$$

By Lemma 6.5, a.s. $S_{m}<S_{m+1}$ if $S_{m}<t$, and for sufficiently large $n S_{n}=t$. For almost all $\omega \in\left\{X_{L^{t}-} \in F\right\}$ there is an $n$ with $S_{n}=L^{t}$. Since $S_{n}$ is previsible, we have by the quasi-left continuity

$$
E\left[g\left(X_{s_{n}}\right) \mid \mathcal{F}\left(s_{n}-\right)\right]=\int \nu\left(X_{s_{n}}, d y\right) g(y)
$$

a.s. on $\left\{S_{n}<t\right\}$ for $g \in \mathcal{E}$. Using a standard argument it follows that

$$
E\left[\phi\left(x_{s_{n}}, s_{n}\right) \mid \mathcal{F}\left(s_{n}-\right)\right]=\int \nu\left(x_{s_{n}-}, d y\right) \phi\left(y, s_{n}\right)
$$

a.s. on $\left\{S_{n}<t\right\}$ for $\phi$ a bounded universally measurable function on the product space $E_{\Delta \times} \times[0, \infty)$.

If $\Lambda \in \mathcal{F}\left(L^{t}-\right)$, then by Lemma $4.1\left(\right.$ b) for each $n$ there exists a set $\Lambda_{n} \epsilon$ $\mathcal{F}\left(s_{n}-\right)$ such that

$$
\left\{X_{L^{t}-} \in F\right\} \cap \Lambda \cap\left\{L^{t}=S_{n}\right\}=\Lambda_{n} \cap\left\{L^{t}=S_{n}\right\} .
$$

Hence, employing (6.1) with $S_{n}=S$ there, we have

$$
\begin{aligned}
E\left[b\left(X_{L^{t}}\right) f\left(X_{t}\right) ; \Lambda, \Phi_{t^{\prime}}, X_{L^{t}-} \in F\right]=\sum_{n=1}^{\infty} E\left[b\left(X_{S_{n}}\right) f\left(X_{t}\right) ; \Lambda_{n}, \Gamma_{S_{n}}, L^{t}=S_{n}\right] \\
=\sum_{n=1}^{\infty} E\left[b\left(X_{S_{n}}\right) E^{X\left(S_{n}\right)}\left[f\left(X_{t-S_{n}}\right) ; T\left(\omega^{\prime}\right)>t-S_{n}\right] ; \Lambda_{n}, \Gamma_{\left.S_{n}\right]}\right. \\
=\sum_{n=1}^{\infty} E\left[\int \nu\left(X_{S_{n}}, d y\right) P^{y}\left(T\left(\omega^{\prime}\right)>t-S_{n}\right) \cdot Q^{b}\left(X_{S_{n},} t-S_{n^{\prime}} f\right) ; \Lambda_{n}, \Gamma_{S_{n}}\right] \\
=\sum_{n=1}^{\infty} E\left[Q^{b}\left(X_{L^{t}-}, t-L^{t}, f\right) ; \Lambda_{n}, \Phi_{t}, S_{n}=L^{t}\right] \\
=E\left[Q^{b}\left(X_{L_{-}^{t}}, t-L^{t}, f\right) ; \Lambda, \Phi_{t}, X_{L^{t}} \in F\right],
\end{aligned}
$$

where the third equality follows by conditioning on $\mathcal{F}\left(S_{n}-\right)$ and the definition of $Q^{b}$.

Case 2. $X_{L^{t}-} \in E_{r}$. The proof is quite similar to that of Case 2 of Theorem 1 , but somewhat simpler because the difficulty of including the information at $L^{t}$ is avoided. Let $\epsilon>0, K$ be a compact subset of $E_{r} \times(0, t)$ with

$$
\left|\bar{Q}^{b}(x, t-s, f)-\bar{Q}^{b}(y, t-r, f)\right|<\epsilon
$$

for all $(x, s)$ and $(y, r)$ in $K$, and 


$$
\Gamma=\left\{0<L^{t}<t,\left(X_{L^{t}-}, L^{t}\right) \in K\right\}-\Psi_{t^{\prime}} .
$$

It suffices to show

$$
\left|E\left[b\left(x_{L^{t}}\right) f\left(x_{t}\right) ; \Lambda, \Gamma\right]-E\left[\bar{Q}^{b}\left(x_{L^{t}}, t-L^{t}, f\right) ; \Lambda, \Gamma\right]\right| \leq \epsilon P(\Lambda \cap \Gamma)
$$

for any $\Lambda \in \mathcal{F}\left(L^{t}-\right)$.

With $R^{\#}$ denoting an ordering of the rationals in $(0, t)$, define, for $(x, s) \in K$ and $s<v \leq t$,

$$
\begin{aligned}
& \bar{u}=\bar{u}_{m}(x, s, v)=\text { first } u \text { in } R^{\#} \text { such that } u<(v-s) \wedge 1 / m \text { and } \\
& \qquad\left|\bar{Q}^{b}(x, t-s, f)-Q^{b}(x, u, t-s, f)\right|<1 / m .
\end{aligned}
$$

The families of stopping times $\left\{S_{a}^{m}\right\}$ and the random times $\left\{U_{a}^{m}\right\}$ are defined exactly as.in the proof of Case 2 of Theorem 1 (from the new $K$ and $\bar{u}_{m}$ ) and possess the same properties, except that since $X_{s_{a}^{m}}$ may be in $A^{i}$, it is not necessarily true that $T \circ \theta_{s_{a}^{m}}=0$ a.s. for $S_{a}^{m}<t$ ( $a$ even). However, this last fact is dispensable. We now define

$$
R_{m}=\left\{\begin{array}{l}
S_{a}^{m} \text { if } a \text { is even and } S_{a}^{m}<L^{t} \leq S_{a+1}^{m}, \\
t \text { otherwise. }
\end{array}\right.
$$

Note that this definition is slightly different from before because $L^{t}=S_{a+1}^{m}$ may occur with positive measure. We claim that

$$
\Gamma \subset \bigcup_{\text {even } \alpha<\beta_{0}}\left\{S_{a}^{m}<L^{t} \leq S_{a+1}^{m}\right\}
$$

except for a set of measure 0 ( $\beta_{0}$ was defined in Case 2 of Theorem 1$)$, which implies

$$
\Gamma \subset\left\{R_{m}<L^{t} \text { for all } m\right\}
$$

except for a set of measure 0 . First for a limit ordinal $a, L^{t} \neq \sup _{\beta<a} S_{\beta}^{m}$ a.s. on $\Gamma$ since $L^{t}$ is totally inaccessible on $\Gamma$ relative to $P$. Next for any even $\alpha$, $\left(X_{u}, u\right) \notin K$ for $u \in\left[\sup _{\beta<\alpha} S_{\beta}^{m}, S_{\alpha}^{m}\right)$; thus, because of Lemma $6.3, L^{t} \not k$

$\left(\sup _{\beta<\alpha} S_{\beta}^{m}, S_{\alpha}^{m}\right]$ almost surely, and (6.4) is established.

The honesty of $R_{m}$ is verified as follows: for $0<u<v \leq t$,

$$
\begin{aligned}
\left\{R_{m}<u\right\} \cap\left\{R_{m}<v\right\}= & \bigcup_{\text {even } a<\beta_{0}}\left\{S_{a}^{m}<u\right\} \\
& \cap\left(\left\{S_{a+2}^{m} \geq v\right\} \cup\left\{S_{a+2}^{m}<v, T \circ \theta_{S_{a+2}^{m}}>v-S_{a+2}^{m}\right\}\right) \\
& \cap\left\{R_{m}<v\right\} .
\end{aligned}
$$


Now fixing $\Lambda \in \mathcal{F}\left(R_{m_{0}}(+)\right)$ and $m \geq m_{0}$ we again use the strong Markov property to obtain

$$
\begin{aligned}
& E\left[b\left(X_{s_{a+1}^{m}}\right) f\left(X_{t}\right) ; \Lambda, s_{a}^{m}<L^{t} \leq S_{a+1}^{m}\right] \\
& \quad=E\left[Q^{b}\left(X_{s_{a}^{m}}, \bar{u}, t-S_{a}^{m}, f\right) ; \Lambda, s_{a}^{m}<L^{t} \leq S_{a+1}^{m}\right]
\end{aligned}
$$

with $\bar{u}=\bar{u}_{m}\left(X_{s_{a}^{m}}, S_{a}^{m}, U_{a}^{m}\right)$. Then summing over $\alpha$ and, as $m \rightarrow \infty$, using the continuity of $b$, equation (6.2) and the definition of $\bar{u}$, we obtain

$$
\begin{aligned}
& \mid E\left[b\left(X_{L^{t}}\right) f\left(X_{t}\right) ; \Lambda, R_{m}<L^{t} \text { for all } m\right] \\
& -E\left[\bar{Q}^{b}\left(X_{\left.L_{-}{ }_{-}, t-L^{t}, f\right)} ; \Lambda, R_{m}<L^{t} \text { for all } m\right] \mid\right. \\
& \leq \epsilon P\left(\Lambda \cap\left\{R_{m}<L^{t} \text { for all } m\right\}\right) .
\end{aligned}
$$

Using Lemma 4.4 we obtain the above inequality for all $\Lambda \in \mathscr{F}\left(L^{t}-\right)$. Since $\Gamma \epsilon$ $\mathcal{F}\left(L^{t_{-}}\right)$, this and (6.5) yield (6.3). Repeating the argument with notational changes yields (6.3) with $\underline{Q}^{b}$ for $\bar{Q}^{b}$ and the proof if complete.

7. The relationship between $Q^{b}$ and $D$. From Theorem 2 we have seen that on $\Omega_{t} Q^{b}\left(X_{L^{t}-}, t-L^{t},.\right)$ can be interpreted as the conditional distribution of the process at $t$ given information up to but not including $L^{t}$. It is reasonable then to expect that $Q^{b}$ can be represented as a weighted average of entrance distributions $D\left(y, t-L^{t},.\right)$ from points accessible from $X_{L^{t}}$. Specifically we show that there exist measures $\eta(x, s, d y)$ such that a.s. on $\Omega_{t}$

$$
Q^{b}\left(X_{L^{t}-}, t-L^{t}, f\right)=\int \eta\left(X_{L_{-}{ }_{-}}, t-L^{t}, d y\right) b(y) D\left(y, t-L^{t}, f\right) .
$$

This will follow from Theorem 2 and (7.4) below.

Definition 7.1. Let $\mathcal{H}$ denote a countable, dense subset of $\mathcal{C}$ with respect to the supnorm and assume the constant function $1 \in \mathcal{H}$. Let

$$
V_{Q}=\bigcap_{b \in \mathcal{H}} W_{Q}(b, 1),
$$

where $W_{Q}(b, f)$ was defined in $\$ 6$.

To shorten our equations we use the notation of

Definition 7.2. When the quantities involved are defined, set

$$
d(x, s, t)=D\left(x, s, H_{t-s} 1\right) \quad \text { and } q(x, s, t)=Q\left(x, s, H_{t-s} 1\right)
$$

where $H_{r} g(x)=E^{x}\left[g\left(X_{r}\right): T>r\right]$.

Suppose that $(x, s) \in V_{Q}$. Then $Q^{b}(x, s, 1)$ can be extended to a bounded linear functional on $\mathcal{C}$, and hence there exists a Borel measure $\eta(x, s, d y)$ such 
that for all $b \in \mathcal{H}$

$$
Q^{b}(x, s, 1)=\int \eta(x, s, d y) b(y) .
$$

Since $Q^{b}(x, s, 1)$ is universally measurable in $(x, s), V_{Q}$ is a universally measurable set in $E_{\Delta} \times(0, \infty)$, and a straightforward argument shows $\eta(x, s, B)$ to be universally measurable in $(x, s)$ for all $B \in \hat{\mathfrak{B}}$. Note that for $x \in E_{b}$

$$
\eta(x, s, d y)=\nu(x, d y) P^{y}(T>s) / \int \nu(x, d z) P^{z}(T>s) .
$$

Theorem 3. Let $b, f \in \hat{\mathcal{G}}$. Then almost surely on $\Omega_{t},\left(X_{L_{-}}, t-L^{t}\right) \in V_{Q}$,

$$
E\left[b\left(X_{L^{t}}\right) \mid \mathcal{F}\left(L^{t}-\right)\right]=\int \eta\left(X_{L^{t}-}, t-L^{t}, d y\right) b(y)
$$

and $\eta\left(X_{L^{t}-}, t-L^{t}, \cdot\right)$ is concentrated on $\left\{y:\left(y, t-L^{t}\right) \in W_{D}(f)\right\} \cap$ $\left(\left\{X_{L^{t}-}\right\} \cup\left\{y: P^{y}\left(T>t-L^{t}\right)>0\right\}\right)$. Furthermore a.s. on $\Omega_{t}$

(7.4) $E\left[b\left(X_{L^{t}}\right) \cdot f\left(X_{t}\right) \mid \mathcal{F}\left(L^{t}-\right)\right]=\int \eta\left(X_{L^{t}}, t-L^{t}, d y\right) b(y) D\left(y, t-L^{t}, f\right)$.

Finally if $0<s<t$ then a.s. on $\Omega_{t} \cap\left\{0<L^{t}<s\right\}$

$$
\eta\left(x_{L_{-} s_{-}} t-L^{t}, d y\right)=\frac{\eta\left(X_{L_{-}}, s-L^{t}, d y\right) d\left(y, s-L^{t}, t-L^{t}\right)}{q\left(X_{\left.L_{-} s_{-}, s-L^{t}, t-L^{t}\right)}\right.} .
$$

Remark. Note that the set of points "accessible" from $X_{L^{t}-}$ depends on $t-L^{t}$ as well as on $X_{L^{t}-}$ itself.

Proof. Since $\mathcal{H}$ is countable, Theorem 2 implies $\left(X_{L^{t}-}, t-L^{t}\right)$ is in $V_{Q}$ and (7.3) holds a.s. on $\Omega_{t}$. Because $L_{t} \in \mathcal{F}\left(L^{t}-\right)$, (7.3) can be extended to

$$
E\left[\phi\left(X_{L^{t}}, L^{t}\right) \mid \mathcal{F}\left(L^{t}-\right)\right]=\int \eta\left(X_{L^{t}}, t-L^{t}, d y\right) \phi\left(y, L^{t}\right)
$$

a.s. on $\Omega_{t}$ for any bounded Borel measurable function $\phi$ on $E_{\Delta} \times(0, \infty)$ and hence to any bounded universally measurable function through the usual argument. The assertion following equation (7.3) then follows from the extension above and the fact that, on $\left\{X_{L^{t}} \neq X_{L^{t}-}\right\}, L^{t}$ can be represented by a countable number of stopping times so that an application of the strong Markov property yields $P^{X\left(L^{t}\right)}\left(T\left(\omega^{t}\right)>t-L^{t}\right)>0$ a.s. on $\Omega_{t} \cap\left\{X_{L^{t}} \neq X_{L^{t}-}\right\}$.

To show (7.4) we apply Theorem 1 to obtain a.s. on $\Omega_{t}$

$$
\begin{aligned}
\left.E\left[b\left(X_{L}\right)\right) f\left(X_{t}\right) \mid \mathcal{F}\left(L^{t}-\right)\right] & =E\left[b\left(X_{L^{t}}\right) D\left(X_{L^{t}}, t-L^{t}, f\right) \mid \mathcal{F}\left(L^{t}-\right)\right] \\
& =\int \eta\left(X_{L^{t}}, t-L^{t}, d y\right) b(y) D\left(y, t-L^{t}, f\right) .
\end{aligned}
$$

For (7.5) let $\Lambda \in \mathfrak{F}\left(L^{s}-\right) \subset \mathfrak{F}\left(L^{t}-\right)$ and simply compute in two different ways to obtain 


$$
\begin{aligned}
& E\left[b\left(X_{L^{t}}\right) ; \Lambda, 0<L^{t}<s\right] \\
& =E\left[Q\left(X_{L_{-} s_{-}}, s-L^{s}, H_{t-s} 1\right) \int \eta\left(X_{L^{s}}, t-L^{s}, d y\right) b(y) ; \Lambda, 0<L^{s}<s\right] \\
& =E\left[\int \eta\left(X_{L_{-}}, s-L^{s}, d y\right) b(y) D\left(y, s-L^{s}, H_{t-s} 1\right) ; \Lambda, 0<L^{s}<s\right] .
\end{aligned}
$$

8. Entrance laws. Let $\left\{L^{t}, 0 \leq t\right\}$ be a coterminal family, $T$ the associated terminal time and $H_{t}$ the semigroup generated by $T$ as given in Definition 7.3.

Definition 8.1. A family of finite measures $\left\{m_{t}, 0<t\right\}$ is called an entrance law relative to $H_{t}$ if for all $f \in \mathcal{C}$

$$
m_{t} \circ H_{s} f=\int m_{t}(d z) H_{s}(z, f)=m_{s+t} f
$$

for all $0<s, t$.

Now the usual proof of the strong Markov property for a stopping time $S$ relies on the fact that the measures $P_{t}\left(X_{S}(\omega), d z\right)=P\left(X_{S+t} \in d z \mid \mathcal{F}(S(+))\right)$ a.s. are part of the original semigroup and are thus trivially entrance laws (relative to the semigroup). Thus to obtain a strong Markov property at $L^{t}$ it is necessary to prove an analogous property for $Q^{b}$ and $D$. As mentioned in the introduction, this is made possible in special cases by means of a normalizing factor and the purpose of this section is to verify that it is possible in general. Since the a:guments involving $D$ are almost identical with those involving $Q^{b}$, we will content ourselves with providing details only for the latter.

The basic ideal underlying this section is that equation (8.5) ought to hold in the limit, and much of our initial effort is directed to defining a subset of $E_{\Delta \times}$ $(0, \infty)$ on which that is true. (See the Corollary to Lemma 8.3.) Once we obtain that we show how the entrance laws relative to $H_{t}$ may be obtained. The next chore is to connect $Q^{b}(x, .,$.$) and D(x, .,$.$) as in \$ 7$, for $x$ in an appropriate subset of $E_{\Delta}$. When we have that we can then prove that $X_{L^{t}}$ and $X_{L^{t}-}$ are in subsets of $E_{\Delta}$ which have all of the desired properties (Theorem 4).

It should be observed that $P^{x}(T>s) \cdot D(x, s,$.$) for x \in A^{i}$ and $\int \nu(x, d y) P^{y}(T>s) \cdot Q^{h}(x, s,$.$) for x \in E_{b}$ are obviously entrance laws with respect to $H_{t}$. However, to avoid making continual qualifications below, we do not bother to exclude these sets in the following discussions.

We begin with

Definition 8.2. For $t$ and $\lambda$ positive rational numbers let $\mathcal{H}_{t, \lambda}=$ $\left\{g: g=H_{t} f_{\lambda}, f \in \mathcal{H}\right\}$, where $\mathcal{H}$ was defined in $\S_{7}$ and where

$$
f_{\lambda}(x)=E^{x}\left[\int_{0}^{T} e^{-\lambda s} f\left(x_{s}\right) d s\right]=\int_{0}^{\infty} e^{-\lambda s} H_{s} f(x) d s .
$$


Let $\mathcal{H}_{t}=\left\{g: g=H_{t} f, f \in \mathcal{H}\right\}$ and

$$
\overline{\mathcal{H}}=\mathcal{H} \cup \bigcup_{0<t, \lambda \text { rational }}\left(\mathcal{H}_{t, \lambda} \cup \mathcal{H}_{t}\right) .
$$

Definition 8.3. For fixed $b \in \mathcal{C}$ let

$$
\bar{W}_{Q}(b)=\bigcap_{f \in \frac{\mathcal{H}}{}}\left(W_{Q}(b, f) \cap W_{Q}(1, f)\right) \text { and } \bar{W}_{D}=\bigcap_{f \in \bar{H}^{\mathcal{H}}} W_{D}(f) \text {. }
$$

Note that since $\overline{\mathcal{H}}$ is countable, $\bar{W}_{Q}(b)$ and $\bar{W}_{D}$ are universally measurable. $\left(W_{Q}(b, f)\right.$ and $W_{D}(f)$ were introduced in Definitions 6.1 and 5.3.)

Now suppose $(x, s) \in \bar{W}_{Q}(b)$. Then $Q^{b}(x, s, \cdot)$ can be extended to a bounded linear functional on $\mathcal{C}$, and thus there exists a Borel measure $Q^{b}(x, s, d z)$ such that

$$
Q^{b}(x, s, f)=\int Q^{b}(x, s, d z) f(z)
$$

for all $f \in \mathcal{H}$. Since $Q^{b}(x, s, f)$ is universally measurable in $(x, s)$ for fixed $f \in \mathcal{H}$, it follows by a standard argument that $Q^{b}(x, s, B)$ is universally measurable for each Borel set $B \in \mathcal{B}$ and then that $\int Q^{h}(x, s, d z) f(z)$ is universally measurable for each $f$ in $\hat{\mathcal{E}}$. Since an analogous argument holds for $D$, the sets $W_{Q}(b)$ and $W_{D}$ defined next are universally measurable in the product space.

Definition 8.4.

$$
\begin{gathered}
W_{Q}(b)=\left\{(x, s) \in \bar{W}_{Q}(b): Q^{b}\left(x, s, A^{r} \cup E_{b}\right)=Q\left(x, s, A^{r} \cup E_{b}\right)=0,\right. \\
\text { and, for all } f \in \overline{\mathcal{H}}, Q^{b}(x, s, f)=\int Q^{b}(x, s, d z) f(z) \\
\text { and } \left.Q(x, s, f)=\int Q(x, s, d z) f(z)\right\} . \\
W_{D}=\left\{(x, s) \in \bar{W}_{D}: D(x, s, f)=\int D(x, s, d z) f(z) \text { for all } f \in \overline{\mathcal{H}}\right. \\
\text { and } \left.D\left(x, s, A^{r} \cup E_{b}\right)=0\right\} .
\end{gathered}
$$

Let $f \in \hat{\mathcal{E}}$. If $(x, s) \in W_{Q}(b)$, redefine $Q^{b}(x, s, f)$ as $\int Q^{b}(x, s, d y) f(y)$, and if $(x, s) \in W_{D}$, redefine $D(x, s, f)$ as $\int D(x, s, d y) f(y)$.

Lemma 8.1. Suppose $r>0$ and $(x, r) \in W_{Q}(b)$. If $s>r$ with $s-r$ rational and $Q\left(x, r, H_{s-r}\right)>0$, then $(x, s) \in W_{Q}(b)$. Moreover

$$
Q^{b}(x, s, g)=Q^{b}\left(x, r, H_{s-r} g\right) / Q\left(x, r, H_{s-r} 1\right)
$$

for all $g \in \hat{\mathcal{E}}$.

Similarly $(x, r) \in W_{D_{\hat{\hat{C}}}^{\prime}} s-r>0$ rational, and $D\left(x, r, H_{s-r} 1\right)>0$ imply $(x, s) \in W_{D}$, and for $g \in \hat{\varepsilon}$

$$
D(x, s, g)=D\left(x, r, H_{s-r} g\right) / D\left(x, r, H_{s-r} 1\right) .
$$

Proof. It suffices to prove (8.2) for $g \in \overline{\mathcal{H}}$ since the remaining assertions 
are immediate. Since $Q\left(x, r, H_{s-r} 1\right)>0$, for sufficiently small rational $u<r$

$$
q_{u}(x, r, s) \equiv Q\left(x, u, r, H_{s-r} 1\right)>0,
$$

and by an application of the Markov property

$$
Q^{h}(x, u, s, g)=Q^{h}\left(x, u, r, H_{s-r} g\right) / q_{u}(x, r, s) .
$$

Since $H_{s-r} \overline{\mathcal{H}} \subset \overline{\mathcal{H}}$, the right-hand side of (8.5) converges to the right-hand side of (8.2), and thus $Q^{b}(x, s, g)$ exists and (8.2) holds for $g \in \overline{\mathcal{H}}$. The same argument holds for $b=1$, and thus $(x, s) \in W_{O}(b)$.

For convenience' sake we will use the notation for all $s>r$

$$
q(x, r, s)=\int Q(x, r, d z) H_{s-r} 1 \text { and } d(x, r, s)=\int D(x, r, d z) H_{s-r} 1
$$

for $(x, r)$ in $W_{Q}(1)$ and $W_{D}$ respectively. By the definition of $W_{Q}(1)$ and $W_{D}$, this notation is consistent with that used in $\$ 7$. Note that both $d(x, r, s)$ and $q(x, r, s)$ are right continuous and decreasing in $s$.

Lemma 8.2. Suppose $(x, r) \in W_{Q}(b)$. Then for all $f \in \mathcal{H}, Q^{b}\left(x, u, r, H_{s} f_{\lambda}\right)$ converges to $\int Q^{b}(x, r, d z) H_{s}\left(z, f_{\lambda}\right)$ as $u \downarrow 0$ for all $s>0$ and rational $\lambda>0$. Furthermore $q_{u}(x, r, s) \rightarrow q(x, r, s)$ for all $s>r$ except possibly those $s$ in

$$
T_{(x, r)}^{Q}=\{s>r: q(x, r, s-)>q(x, r, s)\}
$$

where $q(x, r, s-)=\lim _{u \uparrow s} q(x, r, u)=\int Q(x, r, d z) P^{z}(T \geq s-r)$. Under analogous assumptions, analogous results bold for $D$ and

$$
d_{u}(x, r, s)=D\left(x, u, r, H_{s-r} 1\right) \rightarrow d(x, r, s)
$$

except possibly on

$$
T_{(x, r)}^{D}=\{s>r: d(x, r, s-)>d(x, r, s)\}
$$

where $d(x, r, s-)=\lim _{u \uparrow s} d(x, r, u)=\int D(x, r, d z) P^{z}(T \geq s-r)$.

Proof. If $g$ is uniformly approximable by $g_{n} \in \mathcal{H}$, then $Q^{b}(x, u, r, g) \rightarrow$ $\int Q^{b}(x, r, d z) g(z)$. Since $H_{t} f_{\lambda}$ is uniformly approximable by $H_{s} f_{\lambda}, s$ rational, the first assertion is immediate. For the second assertion note that since both $q_{u}(x, r, s)$ and $q(x, r, s)$ are decreasing in $s$ and $q_{u}(x, r, s) \rightarrow q(x, r, s)$ for $s-r$ rational, we must also have convergence for those $s$ at which $q(x, r, s)$ is continuous.

Parameters of the form $1 / q\left(x, s, s_{0}\right)$. and $1 / d\left(x, s, t_{0}\right)$ turn out to be the appropriate normalizing factors which convert $Q^{b}(x, s, \cdot)$ and $D(x, s, \cdot)$ to entrance laws. The next result provides the key facts for that.

Lemma 8.3. Let $0<r<s$ and suppose both $(x, r)$ and $(x, s)$ are in $W_{Q}(1)$. 
Then if $q(x, r, s)>0$,

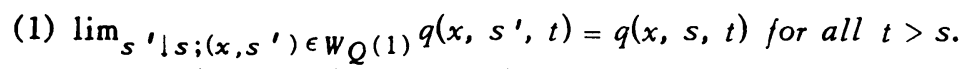

(2) $q(x, r, s) q(x, s, t)=q(x, r, t)$ for all $t>s$.

(3) $q_{u}(x, r, s) \rightarrow q(x, r, s)$ as $u \rightarrow 0$ through rationals.

(4) $T_{(x, s)}^{Q} \subset T_{(x, r)}^{Q}$.

Analogous assertions bold for $d(x, r, s)$.

Proof. Using $g=H_{t-r} 1$ in (8.4) we have for $0<r<s<t$

$$
q_{u}(x, r, s) q_{u}(x, s, t)=q_{u}(x, r, t)
$$

and so $q_{u}(x, r, t)$ is increasing in $r$ for fixed $t$. Hence it suffices to prove (1) for any sequence $s_{n} \downarrow s$, and we may choose $s_{n}<t, s_{n}-s>0$ rational, and $q\left(x, r, s_{n}\right)>0$. Let $t_{m}>t$ be arbitrarily close to $t$ and such that, for all $n$, $q_{u}\left(x, s_{n}, t_{m}\right) \rightarrow q\left(x, s_{n}, t_{m}\right)$ and $q_{u}\left(x, s, t_{m}\right) \rightarrow q\left(x, s_{n}, t_{m}\right)$, a choice made possible by Lemma 8.2. Then using $s, s_{n}$ and $t_{m}$ in (8.6), in the limit, $q\left(x, s, s_{n}\right) q\left(x, s_{n}, t_{m}\right)=q\left(x, s, t_{m}\right)$. Assertion (1) follows by letting $t_{m} \downarrow t$, then $s_{n} \downarrow s$ and finally recalling that $Q\left(x, s, A^{r} \cup E_{b}\right)=0$ or $q(x, s, s+)=1$.

For (2) choose appropriate $r<s<s_{n}<t<t_{m}$, use $r, s_{n}$ and $t_{m}$ in (8.6), and after passing to the limit as $u \rightarrow 0$ through the rationals let $q\left(x, s_{n}, t_{m}\right) \rightarrow$ $q\left(x, s_{n}, t\right) \rightarrow q(x, s, t)$. (3) then follows by (2) and an appropriate choice of $t$ in (8.6), while (4) is immediate from (2).

Corollary. If $(x, r)$ and $(x, s) \in W_{Q}(b)$, then $q(x, r, s)>0$ implies

$$
Q^{b}(x, s, f)=Q^{b}\left(x, r, H_{s-r} f\right) / q(x, r, s)
$$

for any $f \in \hat{\mathcal{G}}$. Similarly if $(x, r)$ and $(x, s) \in W_{D}$ and $d(x, r, s)>0$, then

$$
D(x, s, f)=D\left(x, r, H_{s-r} f\right) / d(x, r, s) \text {. }
$$

Proof. From Lemma 8.2 and Lemma 8.3(3)

$$
Q^{h}\left(x, s, \lambda f_{\lambda}\right)=Q^{b}\left(x, r, H_{s-r} \lambda f_{\lambda}\right) / q(x, r, s)
$$

for $f \in \mathcal{H}$. Letting $\lambda \uparrow \infty$ through the rationals gives (8.7) on $\mathcal{C}$ and thence on $\hat{\mathcal{G}}$.

We now have enough preliminary results to obtain the entrance law property in the form desired.

Definition 8.5. Let $G_{Q}(b)$ denote those $x \in E_{\Delta}$ such that

(i) There exist $s_{n} \downarrow 0$ with $\left(x, s_{n}\right) \in W_{Q}(b)$.

(ii) There exists a $u>0$ such that $q(x, r, s)>0$ for all $0<r<s<u$ and $(x, r) \in W_{Q}(b)$.

$G_{D}$ will denote the analogous set with $D$ and $d$ in lieu of $Q^{b}$ and $q$.

Note that if $x \in G_{Q}(b)$, by Lemma 8.3 


$$
u_{Q}(x)=\sup \{t: q(x, s, t)>0\}
$$

is independent of $s \in(0, u)$, and similarly for

$$
u_{D}(x)=\sup \{t: d(x, s, t)>0\} \text {. }
$$

Lemma 8.4. Suppose $x \in G_{Q}(b)$. Then there exists a family of measures $\widetilde{Q}^{b}(x, s,$.$) and a function \widetilde{q}(x, s, t)$ such that $\widetilde{Q}^{b}$ and $\widetilde{q}$ coincide with $Q^{b}$ and $q$ if $(x, s) \in W_{Q}(b)$ and $0<s<t<u_{Q}(x)$. If $0<s_{0}<u_{Q}(x)$, then $\left\{\widetilde{Q}^{b}(x, s, \cdot) / \widetilde{q}\left(x, s, s_{0}\right), 0<s<\infty\right\}$ is an entrance law relative to $H_{t}$. (Recall the convention $0 / 0=0$.)

If $x \in G_{\widetilde{D}}$, then similarly there exists an extension $\widetilde{D}$ and $\tilde{d}$ such that $\left\{\tilde{D}(x, s, \cdot) / \tilde{d}\left(x, s, t_{0}\right), 0<s<\infty\right\}$ is an entrance law relative to $H_{t}$.

Proof. Let $s_{n} \downarrow 0$ and $\left(x, s_{n}\right) \in W_{Q}(b)$. For all $s>s_{n}$ let

$$
\tilde{Q}^{b}(x, s, f)=Q^{b}\left(x, s_{n}, H_{s-s_{n}} f\right) / q\left(x, s_{n}, s\right)
$$

so that $\tilde{Q}^{b}=Q^{b}$ if $(x, s) \in W_{Q}(b)$ and $s<u_{Q}(x)$. Note that $\widetilde{Q}^{b}(x, s, f)=0$ for all $s \geq u_{Q}(x)$, and that (8.6) and Lemma 8.3 (2) show the definition to be independent of the choice of $s_{n}<s$. Using $\widetilde{q}(x, s, t)=\int \widetilde{Q}(x, s, d z) H_{t-s}(z, 1)$ for $s \leq t$, we extend $\tilde{q}$ to $t<s$ by

$$
\tilde{q}(x, s, t)=\left\{\begin{array}{l}
(\tilde{q}(x, t, s))^{-1} \text { if } t<s<u_{Q}(x), \\
0 \text { if } u_{Q}(x) \leq \max (s, t),
\end{array}\right.
$$

and it is easy to check that for all $0<r, s, t$

$$
\tilde{q}(x, r, s) \tilde{q}(x, s, t)=\tilde{q}(x, r, t) \text {. }
$$

The entrance law property then follows trivially: with $0<s_{0}<u_{Q}(x)$

$$
\frac{\tilde{Q}^{b}\left(x, s, H_{t-s} f\right)}{\tilde{q}\left(x, s, s_{0}\right)}=\frac{Q^{b}\left(x, s_{n}, H_{t-s_{n}} f\right)}{\tilde{q}\left(x, s, s_{0}\right) \tilde{q}\left(x, s_{n}, s\right)}=\frac{\tilde{Q}^{b}(x, t, f)}{\tilde{q}\left(x, t, s_{0}\right)} .
$$

The proof of the assertions for $D$ is completely analogous.

In the sequel we shall be using $\widetilde{Q}(x, s, f), \widetilde{D}(x, s, f), \widetilde{q}(x, s, t)$ and $\tilde{d}(x, s, t)$, but since no confusion should result we suppress the " $\sim$ ". Although these functions are well defined for $x \in G_{Q}(b)$ or for $x \in G_{D}$, we have not yet established their measurability in $(x, s)$, and that is the purpose of the lemma below. Unfortunately the sets $G_{Q}(b)$ and $G_{D}$ themselves need not be universally measurable sets, and we introduce the convention

Definition 8.6. Let $D \subset E_{\Delta}$. Then $g(x)$ is said to be universally measurable on $D$ if the restriction of $g$ to every universally measurable subset of $D$ is universally measurable. A similar convention holds for $D^{*} \subset E_{\Delta} \times(0, \infty)$ and a 
function $g^{*}(x, s)$. $f \in \hat{\mathcal{E}}$ :

Recall first the relations derived above for $0<r<s<t, x \in G_{Q}(b)$ and

$$
q(x, r, s) q(x, s, t)=q(x, r, t), \quad Q^{b}(x, t, f)=Q^{b}\left(x, s, H_{t-s} f\right) / q(x, s, t),
$$

and their counterparts for $x \in G_{D}$

$$
d(x, r, s) d(x, s, t)=d(x, r, t), \quad D(x, t, f)=D\left(x, s, H_{t-s} f\right) / d(x, s, t) .
$$

Then

Lemma 8.5. For fixed $b \in \mathcal{C}$ and $f \in \hat{\mathcal{E}}$ the functions $Q^{b}(x, s, f)$ and $Q(x, s, f)$ are universally measurable on $G_{Q}(b)$ for each $s>0$ and are jointly measurable on $G_{Q}(b) \times(0, \infty)$. In addition $u_{Q}(x)$ is universally measurable on $G_{Q}(b)$. Analogous results bold for $D(x, s, f)$ and $u_{D}(x)$ on $G_{D}$ and $G_{D} \times(0, \infty)$.

Proof. $f$ may be assumed continuous. We shall define universally measurable sets $\hat{G}_{Q}(b) \supset G_{Q}(b)$ as well as universally measurable functions $\hat{Q}^{b}(x, s, f)$ and $\hat{u}_{Q}(x)$ on $\hat{G}_{Q}(b) \times(0, \infty)$ and $\hat{G}_{Q}(1)$ respectively which agree with $Q^{b}$ and $u_{Q}$ on the smaller domains. First for $g \in \mathcal{H}$ and $(x, s) \in E_{\Delta} \times(0, \infty)$ define

$$
\hat{Q}^{h}(x, s, g)=\lim _{\lambda} \lim _{u} Q^{h}\left(x, u, s, \lambda g_{\lambda}\right)
$$

as $u\left\lfloor 0\right.$ and $\lambda \rightarrow \infty$ through the rationals, provided $\lim _{\lambda} \lim _{u} Q(x, u, s, \lambda 1 \lambda)=1$. Let $\hat{W}=\left\{(x, s): \hat{Q}^{b}(x, s, g)\right.$ and $\hat{Q}(x, s, g)=\hat{Q}^{1}(x, s, g)$ exist for all $\left.g . \in \mathcal{H}\right\}$. Then $\hat{W}$ is a jointly universally measurable set, each $s$-section $[\hat{W}]_{s}$ is universally measurable in $E_{\Delta}$, and the functions $\hat{Q}^{b}(x, s, g)$ and $\hat{Q}(x, s, g)$ are jointly universally measurable on $\hat{W}$ and universally measurable on $[\hat{W}]_{s}$ for each fixed $s>0$. Let $\hat{G}_{Q}(b)=\bigcup_{t} \cap_{s<t}[\hat{W}]_{s}$, where $s$ and $t$ range over the positive rationals, and define for $x \in \hat{G}_{Q}(1)$

$$
\hat{u}_{Q}(x)= \begin{cases}\inf \left\{s>0, s \text { rational and } \int \hat{Q}(x, r, d y) H_{s-r} 1(y)>0\right. \\ 0 \text { if }\{\}=\varnothing . & \text { for all small rational } 0<r<s\}\end{cases}
$$

Then $\hat{G}_{Q}(b)$ is universally measurable and $\hat{u}_{Q}$ universally measurable over $\hat{G}_{Q}(1) \supset \hat{G}_{Q}(b)$. If $\hat{Q}^{b}(x, s, f)$ is redefined to be zero for $s \geq \hat{u}_{Q}(x)$, we have $Q^{b}(x, s, f)=\hat{Q}^{b}(x, s, f)$ on $G_{Q}(b) \times(0, \infty)$ and $u_{Q}=\hat{u}_{Q}$ on $G_{Q}(b)$. The first equality follows from the fact that for $x \in G_{Q}(b)$ and $s<u_{Q}(x)$

$$
\frac{Q^{b}\left(x, u, s, \lambda f_{\lambda}\right)}{Q\left(x, u, s, \lambda 1_{\lambda}\right)}=\frac{Q^{b}\left(x, u, r, H_{s-r} \lambda f_{\lambda}\right)}{Q\left(x, u, r, H_{s-r} \lambda 1_{\lambda}\right)} \rightarrow \frac{Q^{b}\left(x, r, H_{s-r} f\right)}{q(x, r, s)}
$$

as $u \rightarrow 0$ and $\lambda \rightarrow \infty$ through the rationals, assuming $r<s$ and $(x, r) \in W_{Q}(b)$. 
The second equality follows from the definition of $u_{Q}$ and $\hat{u}_{Q}$.

The proof for $D$ and $u_{D}$ is similar.

Next we connect the entrance laws $Q^{b}(x, s,$.$) and D(x, s, \cdot)$. Let $G_{Q}=$ $\bigcap_{b \in \mathcal{H}} G_{Q}(b)$. Then, as before, for each $x \in G_{Q}$ and $s>0, Q^{b}(x, s, 1)$ defines a linear functional on $\mathcal{C}$ and so there exists a measure $\eta(x, s, \cdot)$ on $E_{\Delta}$ with $Q^{b}(x, s, 1)=\int \eta(x, s, d y) b(y)$. Clearly $\eta\left(x, s, E_{\Delta}\right)=1$ if $s<u_{Q}(x)$ and is zero otherwise. For $s<u_{Q}(x)$ the definition extends that of $\$ 7$. By virtue of the above lemma, for $B \in \hat{\Re}, \eta(x, s, B)$ is jointly universally measurable on $G_{Q} \times$ $(0, \infty)$ and universally measurable on $G_{Q}$ for each $s>0$.

Definition 8.7. Let $H_{Q}$ be those $x \in G_{Q}$ such that for some sequence $s_{n} \downarrow 0$

(i) $\eta\left(x, s_{n}, \cdot\right)$ is concentrated on $\{x\} \cup A^{i}$.

(ii) $\eta\left(x, s_{n}, \cdot\right)$ is concentrated on $G_{D}$, that is on some Borel set $B \subset G_{D}$.

(iii) For all $h, f \in \mathcal{C}$

$$
Q^{b}\left(x, s_{n}, f\right)=\int \eta\left(x, s_{n}, d y\right) b(y) D\left(y, s_{n}, f\right) .
$$

It will be clear from the proof of the following lemma that the sequence $s_{n}$ in Definition 8.7 may be fixed independently of $x \in H_{Q}$.

Lemma 8.6. Suppose $x \in H_{Q}, 0<r<s, b \in \mathcal{C}$ and $f \in \hat{\mathcal{G}}_{\text {. Then }}$

(1) $\eta(x, s,$.$) is concentrated on \left(\{x\} \cup A^{i}\right) \cap G_{D}$.

(2) $Q^{b}(x, s, f)=\int \eta(x, s, d y) b(y) D(y, s, f)$.

(3) $Q^{h}(x, s, f)=\int \eta(x, r, d y) b(y) D\left(y, r, H_{s-r} f\right) / q(x, r, s)$, in particular $\eta(x, s, d y)=\eta(x, r, d y) d(y, r, s) / q(x, r, s)$.

(4) $q(x, r, s)=\int \eta(x, r, d y) d(y, r, s)$.

Proof. Let $s_{n}<s$ be as in Definition 8.6. Then for all $b \in \mathcal{C}, f \in \hat{\mathcal{E}}$,

$$
Q^{b}(x, s, f)=\frac{Q^{b}\left(x, s_{n}, H_{s-s_{n}} f\right)}{q\left(x, s_{n}, s\right)}=\frac{\int \eta\left(x, s_{n}, d y\right) b(y) D\left(y, s_{n}, H_{s-s_{n}} f\right)}{q\left(x, s_{n}, s\right)} .
$$

With $f=1$ we get $\eta(x, s, d y)=\eta\left(x, s_{n}, d y\right) d\left(y, s_{n}, s\right) / q\left(x, s_{n}, s\right)$ and (1) immediately follows. (2) also follows since

$$
D(y, s, f)=D\left(y, s_{n}, H_{s-s_{n}} f\right) / d\left(y, s_{n}, s\right)
$$

If $s_{n}<r<s$, then 


$$
\begin{aligned}
Q^{b}(x, s, f) & =\frac{\int \eta\left(x, s_{n}, d y\right) b(y) D\left(y, s_{n}, H_{r-s_{n}} H_{s-r} f\right)}{q\left(x, s_{n}, s\right)} \\
& =\frac{\int \eta\left(x, s_{n}, d y\right) b(y) d\left(y, s_{n}, r\right) D\left(y, r, H_{s-r} f\right)}{q\left(x, s_{n}, r\right) q(x, r, s)} \\
& =\frac{\int \eta(x, r, d y) b(y) D\left(y, r, H_{s-r} f\right)}{q(x, r, s)}
\end{aligned}
$$

in view of the foregoing; (3) is thus established, and (4) follows by setting $f=$ $b=1$.

We can now prove

Theorem 4. For each initial measure $\mu$ and $t>0$

(i) $X_{L t} \in G_{D}$ (i.e. in a Borel subset of $G_{D}$ ) a.s. on $\left\{L^{t}<t\right\}$, and

(ii) $X_{L^{t}-} \in H_{Q}$ a.s. on $\Omega_{t}$.

Proof. Fix $\mu$ and $t>0$. We only prove (2) and (1) is similar (and simpler). Now it follows from Theorem 2 that, for each $r>0,\left(X_{L^{r}-}, r-L^{r}\right) \in W_{Q}(b, f)$ and

$$
E\left[b\left(x_{L^{r}}\right) f\left(x_{r}\right) \mid \mathcal{F}\left(L^{r}-\right)\right]=Q^{b}\left(X_{L^{r}}, r-L^{r}, f\right)
$$

a.s. on $\Omega_{r}$ for all $f \in \overline{\mathcal{H}}$ and $b \in \mathcal{H}$. We obtain the measures $Q^{b}\left(X_{L^{r}-{ }^{\prime}}{ }^{-} L^{r},.\right)$ as $f$ runs through $\mathcal{H}$ and see that the integrals of $f \in \overline{\mathcal{H}}$ against these measures are the $Q^{b}\left(X_{L^{r}}, r-L^{r}, f\right)$ above, a.s. on $\Omega_{r}$. It is also immediate that $Q^{b}\left(X_{L_{-}-}, r-L^{r},.\right)$ is concentrated on $A^{i}$ a.s. on $\Omega_{r}$, and thus up to a set of $P^{\mu}$ measure zero on $\Omega_{r}$ we have $\left(X_{L^{r}-}, r-L^{r}\right) \in W_{Q}(b)$ for all $b \in \mathcal{H}$. Also if $r<t$ the following computation implies that $q\left(X_{L^{r}{ }^{\prime}}, r-L^{r}, t-L^{r}\right)>0$ a.s. on $\Omega_{t} \cap\left\{L^{t}<r\right\} \subset \Omega_{r}$ :

$$
\begin{aligned}
& \left.E 1_{\left\{L^{t}<r\right\}} \mid \mathcal{F}\left(L^{r}-\right)\right]=E\left[E\left(1_{\left\{T_{\circ} \theta_{r}>t-r\right.} \mid \mathcal{F}_{r}\right) \mid \mathcal{F}\left(L^{r}-\right)\right] \\
& =E\left[H_{t-r} 1\left(X_{r}\right) \mid \mathcal{F}\left(L^{r}-\right)\right]=Q\left(X_{L^{r}-r}, r-L^{r}, H_{t-r} 1\right) \\
& =q\left(X_{L^{r}}, r-L^{r}, t-L^{r}\right)
\end{aligned}
$$

a.s. on $\Omega_{r}$. Thus for $0<r<t$ there is a $\sigma$-compact set $J, \subset \bigcap_{b \in \mathcal{H}^{(}}\left(W_{Q}(b)\right) \cap$ $\left(E_{\Delta} \times(0, r)\right)$ such that $q(x, s, t-r+s)>0$ for all $(x, s) \in J_{r}$ and such that $\left(X_{L^{r}-}, r-L^{r}\right) \in J_{r}$ a.s. on $\Omega_{t} \cap\left\{L^{t}<r\right\}$. It then follows that $C_{n, r}=$ $\left\{x:(x, s) \in J_{r}\right.$, some $s$ in $\left.[r-1 / n, r)\right\}$ is $\sigma$-compact and $B_{t}(\mu, Q)=$ $\bigcap_{n=1}^{\infty} \cup_{0<r<t, r \text { sational }} C_{n, r}$ is a Borel subset of $E_{\Delta}$. By the construction $B_{t}(\mu, Q) \subset$ 
$G_{Q}$, and $X_{L^{t_{-}}} \in B_{t}(\mu, Q)$ a.s. on $\Omega_{t^{*}}$

Proceeding in a similar manner using Theorem 1 we can find $B_{t}(\mu, D) \subset G_{D}$ such that $X_{L^{t}} \in B_{t}(\mu, D) \subset G_{D}$ a.s. $P^{\mu}$ on $\left\{L^{t}<t\right\}$. To complete the proof of part (ii) we define $\sigma$-compact sets $J_{r}^{\prime} \subset J_{r}$ by imposing the additional condition that, for $(x, s) \in J_{r}^{\prime}, \eta(x, s,$.$) is concentrated on \left(\{x\} \cup A^{i}\right) \cap G_{D}$ and satisfies $Q^{b}(x, s, f)=\int \eta(x, s, d y) b(y) D(y, s, f)$ for all $f, b \in C$. By virtue of Theorem 3 , it follows that, a.s. on $\Omega_{r},\left(X_{L^{r}}, r-L^{r}\right) \in J_{r}^{\prime}$. Then define $C_{n, r}^{\prime}$ from $J_{r}^{\prime}$ and $B_{t}^{\prime}(\mu, Q)$ from the $C_{n, r}^{\prime}$ as before to obtain, a.s. on $\Omega_{t}, X_{L^{t}-} \epsilon B_{t}^{\prime} \epsilon$ $B_{t}^{\prime}(\mu, Q) \subset H_{Q}$

9. The strong Markov property at $L^{t}$. As mentioned in the introduction, the evolution of the process from $L^{t}$ may depend not only on $X_{L^{t}}$ (or $X_{L^{t}}$ ) but also on $t-L^{t}$. In this section we obtain the explicit dependence on these parameters, as usual confining our attention in detail to only one case.

Theorem 5. Let $f_{i} \in \hat{\mathcal{E}}, 1 \leq i \leq n+1$ and $0<s_{1}<s_{2}<\ldots<s_{n}<t$ with $\bar{t}=t-L^{t}$. Then a.s. on $\left\{0<L^{t}<\bar{t}-s_{n}\right\}$

$$
\begin{aligned}
& E\left[f_{n+1}\left(X_{t}\right) \prod_{k=1}^{n} f_{k}\left(X_{L^{t}+s_{k}}\right) \mid \mathcal{F}\left(L^{t}\right)\right] \\
& =\int \cdots \int \frac{D\left(X L^{t}, s_{1}, d z_{1}\right)}{d\left(X_{L^{t}}, s_{1}, \bar{t}\right)} f_{1}\left(z_{1}\right) H_{s_{2}-s_{1}}\left(z_{1}, d z_{2}\right) \\
& \quad \ldots \cdot f_{n-1}\left(z_{n-1}\right) H_{s_{n}-s_{n-1}}\left(z_{n-1}, d z_{n}\right) f_{n}\left(z_{n}\right) H_{\bar{t}-s_{n}}\left(z_{n}, f_{n+1}\right) .
\end{aligned}
$$

If $n=1$ and $f_{2}=1$, using the convention $g_{s}(x)=P^{x}(T>s)$, the foregoing takes the form

$$
E\left[f\left(X_{L^{t}+s}\right) \mid \mathcal{F}\left(L^{t}\right)\right]=\frac{D\left(X_{L^{t}}, s, f g_{\bar{t}-s}\right)}{D\left(X_{L^{t}}, s, g_{\bar{t}-s}\right)}
$$

a.s. on $\left\{0<L^{t}<t-s\right\}$.

Almost surely on $\left\{0<L^{t}<t-s_{n}\right\} \cap \Omega_{t}$, if $b \in \mathcal{C}$, 


$$
\begin{aligned}
& E\left[b\left(X_{L^{t}}\right) f_{n+1}\left(X_{t}\right) \prod_{k=1}^{n} f_{k}\left(X_{L^{t}+s_{k}}\right) \mid \mathcal{F}\left(L^{t}-\right)\right] \\
& =\int \cdots \int \frac{Q^{b}\left(X_{L^{t}-}, s_{1}, d z_{1}\right)}{q\left(X_{L^{t}}, s_{1}, \bar{t}\right)} f_{1}\left(z_{1}\right) \\
& \quad \ldots \cdot H_{s_{n}-s_{n-1}}\left(z_{n-1}, d z_{n}\right) f_{n}\left(z_{n}\right) H_{\bar{t}-s_{n}}\left(z_{n}, f_{n+1}\right), \\
& =\int \cdots \iint \frac{\left.\eta_{L^{t}-}, s_{1}, d y\right)}{q\left(X_{\left.L_{-}^{t}, s_{1}, \bar{t}\right)} b(y) D\left(y, s_{1}, d z_{1}\right) f_{1}\left(z_{1}\right)\right.} \\
& \cdot \ldots \cdot H_{s_{n}-s_{n-1}}\left(z_{n-1}, d z_{n}\right) f_{n}\left(z_{n}\right) H_{\bar{t}-s_{n}}\left(z_{n}, f_{n+1}\right)
\end{aligned}
$$

and

$$
\left.E\left[b\left(X_{L^{t}}\right) f\left(X_{L^{t}+s}\right) \mid \mathcal{F}\left(L^{t}-\right)\right]=Q^{b}\left(X_{L^{t}-}, s, f g_{\bar{t}-s}\right) / Q_{L^{t}-}, s, g_{\bar{t}-s}\right)
$$

a.s. on $\left\{0<L^{t}<t-s\right\} \cap \Omega_{t}$.

Remark. By virtue of the first part of the theorem we also have a strong Markov property relative to $\mathcal{F}\left(L^{t_{-}}\right)$on the set $\Psi_{\ell}$ : simply replace $q$ by $d$ and $Q^{b}\left(X_{L_{t_{-}}}, \cdot, \cdot\right)$ by $b\left(X_{L_{t_{-}}}\right) D\left(X_{L_{t_{-}}}, \cdot, \cdot\right)$ in the equations above.

Proof. We confine our attention to $Q^{b}$ and also assume that all the $f_{i}$ are in $C$. By virtue of the preceding section we can restrict $Q^{b}(x, s, f)$ to $B_{t}(\mu, Q) \times(0, \infty)$, thereby guaranteeing a universally measurable function in $(x, s)$. Finally, let $n=2$ to simplify the notation.

Since

$$
\left\{0<L^{t}<t-s_{2}\right\}=\lim _{N \rightarrow \infty}\left\{0<L^{t-s_{2}}<t-s_{2}, T \circ \theta_{t-s_{2}}>s_{2}+1 / N\right\}
$$

it suffices to consider the limit of

$$
E\left[b\left(X_{L^{t}}\right) f_{1}\left(X_{L^{t}+s_{1}}\right) f_{2}\left(X_{L^{t}+s_{2}}\right) f_{3}\left(X_{t}\right)\right.
$$

$$
\left.\Lambda, 0<L^{t-s_{2}}<t-s_{2}, T \circ \theta_{t-s_{2}}>s_{2}+1 / N\right]
$$

where $\Lambda \in \mathcal{F}\left(L^{t}-\right)$. With $u_{k}=k 2^{-m}\left(t-s_{2}\right), 0 \leq k \leq 2^{m}$, we can write $(9.1)$ as

$$
\begin{aligned}
& \lim _{m \rightarrow \infty} \sum_{k=2}^{m} E\left[b\left(x_{L}^{t-s_{2}}\right) f_{1}\left(X_{u_{k}+s_{1}}\right) f_{2}\left(X_{u_{k}+s_{2}}\right) f_{3}\left(X_{t-u_{k-1}+u_{k}}\right)\right. \\
& \left.\Lambda, u_{k-1} \leq L^{t-s_{2}}<u_{k}, T \circ \theta_{t-s_{2}}>s_{2}+1 / N\right] .
\end{aligned}
$$


Since $\mathfrak{F}^{t}\left(L^{t}\right) \cap\left\{L^{t}=L^{u}\right\}=\mathcal{F}\left(L^{u}-\right) \cap\left\{L^{t}=L^{u}\right\}$, we can choose $\Lambda_{k} \in \mathcal{F}\left(L^{u_{k}}\right)$ in place of $\Lambda$ in each term in the preceding, and the $k$ th term is then

$$
\begin{array}{r}
E\left[b\left(X_{L} u_{k}\right) E^{X}{ }^{u_{k}\left[f_{1}\right.}\left(X_{s_{1}}\right) f_{2}\left(X_{s_{2}}\right) f_{3}\left(X_{t-u_{k-1}}\right) ; T>t-u_{k}+1 / N\right] ; \\
\left.\Lambda_{k}, u_{k-1} \leq L^{u_{k}}<u_{k}\right] \\
=E\left[Q^{b}\left(X_{L}{ }^{u_{k}}, u_{k}-L^{u_{k}}, H_{s_{1}} g_{k}\right) ; \Lambda_{k}, u_{k-1} \leq L^{u_{k}}<u_{k}\right]
\end{array}
$$

where

$$
g_{k}(z)=f_{1}(z) E^{z}\left[f_{2}\left(X_{s_{2}-s_{1}}\right) f_{3}\left(X_{t-u_{k-1}-s_{1}}\right) ; T>t-u_{k}-s_{1}+1 / N\right] .
$$

If $y=X_{L} u_{k}$ and $v_{k}=u_{k}-L^{u_{k}}$, we have a.s. on $\Omega_{u_{k}}$,

$$
\frac{Q^{h}\left(y, v_{k}, H_{s_{1}} g_{k}\right)}{q\left(y, v_{k}, t-u_{k}+1 / N\right)}=\frac{Q^{b}\left(y, s_{1}, H_{v_{k}} g_{k}\right)}{q\left(y, s_{1}, t-u_{k}+1 / N\right)} .
$$

Multiplying and dividing by $q\left(y, v_{k}, t-u_{k}+1 / N\right)$, we thus wish to evaluate

$$
\begin{aligned}
& \lim _{N} \lim _{m} \sum_{k=2}^{2^{m}} E\left[\frac{Q^{b}\left(X_{L^{t}-}, s_{1}, H_{v_{k}} g_{k}\right)}{q\left(X_{L^{t}-}, s_{1}, t-L^{t}-v_{k}+1 / N\right)} ;\right. \\
& \left.\Lambda, u_{k-1} \leq L^{t}<u_{k}, T \circ \theta_{t}>1 / N\right] .
\end{aligned}
$$

It is easy to see that this is

$$
E\left[\frac{Q^{b}\left(X_{L^{t}}, s_{1}, f_{1} \cdot H_{s_{2}-s_{1}}\left(f_{2} H_{\bar{t}_{-} s_{2}} f_{3}\right)\right)}{q\left(X_{L^{t}}, s_{1}, t\right)} ; \Lambda, 0<L^{t}<t-s_{2}\right] .
$$

The last assertion then follows from Theorem 4, while an analogous argument for $D$ completes the proof.

10. The strong Markov property at coterminal times. So far we have concentrated on a given coterminal family $\left\{L^{t}, t \geq 0\right\}$. If these times arise from an exact coterminal time $L$ as defined in $\$ 3$, then all of the foregoing results carry over easily to $L$. This may be shown either by proceeding directly and repeating the proofs of Theorems 1 through 4 for $L$ or else by extending the results from $\left\{L^{t}, 0 \leq t\right\}$ to $L$, assuming $L=\lim _{t \rightarrow \infty} L^{t}$. To shorten our discussion we take the latter approach. 
Definition 10.1. If $(x, s) \in W_{Q}(b)$ and $q(x, s, \infty)=\lim _{t \rightarrow \infty} q(x, s, t)>0$, we say that $Q_{L}^{b}(x, s, f)$ exists and define

$$
Q_{L}^{b}(x, s, f)=\frac{Q^{h}\left(x, s, f g_{\infty}\right)}{q(x, s, \infty)}=\frac{Q^{h}\left(x, s, f g_{\infty}\right)}{Q\left(x, s, g_{\infty}\right)},
$$

where $g_{\infty}(x)=P^{x}(T=\infty)$.

If $(x, s) \in W_{D}$ with $d(x, s, \infty)>0$, we say that $D_{L}(x, s, f)$ exists and

$$
D_{L}(x, s, f)=\frac{D\left(x, s, f g_{\infty}\right)}{d(x, s, \infty)}=\frac{D\left(x, s, f g_{\infty}\right)}{D\left(x, s, g_{\infty}\right)} .
$$

Theorem 6. Let $f \in \hat{\mathcal{E}}$ and $b \in \mathcal{C}$.

(i) Almost surely on $\{L<t\}, D_{L}\left(X_{L}, t-L, f\right)$ is defined and

$$
E\left[f\left(X_{t}\right) \mid \mathcal{F}(L)\right]=D_{L}\left(X_{L}, t-L, f\right) .
$$

(ii) Almost surely on $\Omega_{t} \cap\{L<t\}, Q_{L}^{b}\left(X_{L-}, t-L, f\right)$ is defined and

$$
E\left[b\left(X_{L}\right) f\left(X_{t}\right) \mid \mathcal{F}\left(L_{-}\right)\right]=Q_{L}^{b}\left(X_{L-}, t-L, f\right) .
$$

Almost surely on $\{L<t\} \cap \Psi_{t}$,

$$
E\left[b\left(X_{L}\right) f\left(X_{t}\right) \mid \mathcal{F}\left(L_{-}\right)\right]=b\left(X_{L_{-}}\right) D\left(X_{L_{-}}, t-L, f\right) .
$$

Remark. If the property of nearly quasi-left continuity as given in (2.2) is weakened to hold only on $\left\{T_{n}<T=\lim _{m} T_{m}<\zeta\right\}$, the last two results above as well as the corresponding results in Theorems 7 and 8 will hold as stated, provided the conditions $L<t$ and $L<\infty$ are replaced by $L<t \wedge \zeta$ and $L<\zeta$. See the remark following the statement of Theorem 2.

Proof. The argument consists of reducing $L$ to $L^{t}$ and using our earlier results. Since $L=L^{t}$ on $\{L<t\}, \mathcal{F}\left(L_{-}\right) \cap\{L<t\}=\mathcal{F}\left(L^{t}-\right) \cap\{L<t\}$, and we let $\Lambda_{t} \in \mathfrak{F}\left(L^{t}-\right)$ correspond to a given $\Lambda \in \mathfrak{F}\left(L_{-}\right)$. Then

$$
\begin{gathered}
E\left[b\left(X_{L}\right) f\left(X_{t}\right) ; \Lambda, \Omega_{t^{\prime}} L<t\right]=E\left[b\left(X_{L^{t}}\right) f\left(X_{t}\right) g_{\infty}\left(X_{t}\right) ; \Lambda_{t}, \Omega_{t}\right] \\
\quad=E\left[Q_{L}^{b}\left(X_{L^{t}-}, t-L^{t}, f\right) \cdot q\left(X_{L^{t}-}, t-L^{t}, \infty\right) ; \Lambda_{t}, \Omega_{t}\right] \\
=E\left[Q_{L}^{b}\left(X_{L_{-}}, t-L, f\right) ; \Lambda, \Omega_{t}, L<t\right] .
\end{gathered}
$$

To carry over the other results of the earlier sections we concentrate on the set $\{L<\infty\}$ rather than the sets $\{L<t\}$. Define

$$
\Omega_{\infty}=\bigcup_{0<t \text { rational }} \Omega_{t} \cap\{L<t\} .
$$

It is easy to see that $\Omega_{\infty} \cap\left\{0<L<\infty, X_{L-} \in E_{r}\right\}$ is the subset of $\{0<L<\infty\}$ with the largest measure on which $L$ is totally inaccessible relative to $P$. Similarly $\Phi_{\infty}=\Omega_{\infty} \cap\left\{0<L<\infty, X_{L-} \in E_{b}\right\}$ is the subset of $\{0<L<\infty$, 
$\left.X_{L-} \epsilon E_{b}\right\}$ with the largest measure satisfying the property that for any previsible stopping time $S$ there exist $\Gamma_{S} \in \mathcal{F}(S-)$ such that $\Phi_{\infty} \cap\{L=S\}=\Gamma_{S} \cap$ $\left\{T \circ \theta_{S}=\infty\right\}$. Note that both $\Phi_{\infty}$ and $\Omega_{\infty}$ are in $\mathcal{F}\left(L_{-}\right)$.

Definition 10.2.

$$
\begin{aligned}
& G_{D}^{L}=G_{D} \cap\left\{u_{D}=\infty\right\} \cap\{d(x, 1, \infty)>0\}, \\
& G_{Q}^{L}=G_{Q} \cap\left\{u_{Q}=\infty\right\} \cap\{q(x, 1, \infty)>0\}, \\
& H_{Q}^{L}=H_{Q} \cap G_{Q}^{L} .
\end{aligned}
$$

With $g_{\infty}(x)=P^{x}(T=\infty)$, let $K_{t}(x, f)=H_{t}\left(x, f g_{\infty}\right) / g_{\infty}(x)$ and note that $K_{t}$ is a semigroup on $\left\{g_{\infty}>0\right\}$. Finally, for $x \in H_{Q}^{L}$ and $0<s<\infty$ let

$$
\eta(x, d y)=\eta(x, s, d y) d(y, s, \infty) / q(x, s, \infty)
$$

and note $\eta(x, d y)$ is independent of $s$ (see Lemma 8.6).

Theorem 7. (1) For eacb $x \in G_{Q}^{L},\left\{Q_{L}^{b}(x, s, \cdot), 0<s<\infty\right\}$ is an entrance law relative to $K_{t}$, and, for each $x \in G_{D}^{L},\left\{D_{L}(x, s,),. 0<s<\infty\right\}$ is also an entrance law relative to $K_{i}$.

(2) For $x \in H_{Q}^{L}, \eta(x, d y)$ is a probability measure concentrated on $\left(\{x\} \cup\left\{y: g_{\infty}(y)>0\right\}\right) \cap G_{D}^{L}$ and satisfies

$$
Q_{L}^{h}(x, s, f)=\int \eta(x, d y) b(y) D_{L}(y, s, f)
$$

for $b \in \mathcal{C}, f \in \hat{\mathcal{E}}$ and $s>0$.

(3) For any initial measure $\mu, X_{L} \in G_{D}^{L}$ a.s. on $\{L<\infty\}$, and $X_{L-} \in H_{Q}^{L}$ a.s. on $\Omega_{\infty}$.

Proof. The verification of (1) is a triviality; e.g.

$$
\begin{aligned}
Q_{L}^{b}\left(x, s, K_{t} f\right) & =\frac{Q^{b}\left(x, s, H_{t}\left(f g_{\infty}\right)\right)}{q(x, s, \infty)}=\frac{Q^{b}\left(x, s+t, f g_{\infty}\right)}{q(x, s+t, \infty)} \\
& =Q_{L}^{b}(x, s+t, f) .
\end{aligned}
$$

By (1) of Lemma 8.6 and the fact that $g_{\infty}(y)>0$ if $y \in A^{i}$ and $d(y, s, \infty)>0$ for any $s>0, \eta(x, \cdot)$ is concentrated on $\left(\{x\} \cup\left\{y: g_{\infty}(y)>0\right\}\right) \cap G_{D^{*}}^{L}$ By (3) of Lemma 8.6

$$
\begin{aligned}
Q_{L}^{b}(x, s, f) & =\frac{Q^{h}\left(x, s, f g_{\infty}\right)}{q(x, s, \infty)}=\frac{\int \dot{\eta}(x, s, d y) b(y) D\left(y, s, f g_{\infty}\right)}{q(x, s, \infty)} \\
& =\int \eta(x, d y) b(y) D_{L}(y, s, f),
\end{aligned}
$$

and with $f=b=1$ we see that $\eta(x, \cdot)$ has total mass equal to 1 . It remains to prove (3). Since $L=L^{t}$ a.s. on $\{L<t\}$, Theorem 4 clearly implies $X_{L} \in G_{D}$ 
a.s. on $\{L<\infty\}$ and $X_{L-} \in H_{Q}$ a.s. on $\Omega_{\infty}$. To complete the proof it suffices to show $d\left(X_{L}, r, \infty\right)>0\left(q\left(X_{L-}, r, \infty\right)>0\right)$ for some small $r$ a.s. on $\{L<\infty\}$ (on $\Omega_{\infty}$ ). But by a computation similar to (8.12) we have, for each rational $t$, $d\left(X_{L}, t-L, \infty\right)>0$ a.s. on $\{L<t\}$ and $q\left(X_{L-}, t-L, \infty\right)>0$ a.s. on $\Omega_{\infty} \cap$ $\{L<t\}$.

Remark. As the reader may have noticed, we have spent quite a bit of effort working around the fact that $G_{Q}$ and $G_{D}$ (and hence $G_{Q}^{L}$ and $G_{D}^{L}$ ) need not be universally measurable. This difficulty arose because we were forced to deal with times of the form $t-L^{t}$. However, in a direct proof of our results for $L$, the difficulty is circumvented by simply dealing with times of the form $L+s$. It turns out that the sets corresponding to $G_{Q}^{L}$ and $G_{D}^{L}$ will then be universally measurable.

As our last result we have that the given process possesses a strong Markov property at $L$ in the following sense: the post $L$ process conditioned on the past proceeds according to the new stationary transition probabilities $K_{t}$ with an initial distribution of $Q_{L}$. An analogous description holds for conditioning on $\mathcal{F}(L)$ and initial distributions $D_{L}$. The proof reduces to an application of Theorem 5 using now familiar details.

Theorem 8. (i) Let $f_{i} \in \hat{\mathcal{E}}, 1 \leq i \leq n$ and $0<s_{1}<\cdots<s_{n}$. Then almost surely on $\{L<\infty\}$

(ii) If $b \in \mathcal{C}$, then a.s. on $\Omega_{\infty}$

$$
\begin{array}{r}
E\left[\prod_{k=1}^{n} f_{k}\left(X_{L+s_{k}}\right) \mid \mathcal{F}(L)\right] \\
=\int \cdots \int D_{L}\left(X_{L}, s_{1}, d z_{1}\right) f_{1}\left(z_{1}\right) K_{s_{2}-s_{1}}\left(z_{1}, d z_{2}\right) \\
\quad \ldots \cdot f_{n-1}\left(z_{n-1}\right) K_{s_{n}-s_{n-1}}\left(z_{n-1}, f_{n}\right) .
\end{array}
$$

$$
\begin{gathered}
E\left[b\left(X_{L}\right) \prod_{k=1}^{n} f_{k}\left(X_{L+s_{k}}\right) \mid \mathcal{F}(L-)\right] \\
=\int \cdots \int Q_{L}^{b}\left(X_{L-}, s_{1}, d z_{1}\right) f_{1}\left(z_{1}\right) K_{s_{2}-s_{1}}\left(z_{1}, d z_{2}\right) \\
\cdot \ldots \cdot f_{n-1}\left(z_{n-1}\right) K_{s_{n-s_{n-1}}}\left(z_{n-1}, f_{n}\right) \\
=\int \cdots \int \eta\left(X_{L-}, d y\right) b(y) D_{L}\left(y, s_{1}, d z_{1}\right) f_{1}\left(z_{1}\right) K_{s_{2}-s_{1}}\left(z_{1}, d z_{2}\right) \\
\quad \ldots \cdot f_{n-1}\left(z_{n-1}\right) K_{s_{n}-s_{n-1}}\left(z_{n-1}, f_{2}\right) .
\end{gathered}
$$

Also, if we define $\Psi_{\infty}$ as $\left\{0<L<\infty, X_{L-} \in A^{i}\right\}-\Omega_{\infty}$ the first equality of (ii) bolds on $\Psi_{\infty}$ provided $Q_{L}^{b}\left(X_{L_{-}}, \cdot, \cdot\right)$ is replaced by $b\left(X_{L_{-}}\right) D\left(X_{L_{-}}, \cdot, \cdot\right)$. 
11. An example of nonexistence of $Q(x, s, \cdot)$ and $D(x, s, \cdot)$. Throughout this paper we have been careful to prove the existence of the conditional distributions $Q$ and $D$, and the purpose of this section is to show that such care is necessary. Specifically, we give an example of a process and a coterminal family $\left\{L^{t}, t \geq 0\right\}$ such that, for $x=0, Q(0, s,$.$) and D(0, s,$.$) do not exist for$ all $s>0$.

Let $E_{\Delta}=[0,1] \cup\{2\}$ and the process be defined as follows: the states $\{1\}$ and $\{2\}$ are absorbing and starting from $0 \leq x<1$ a particle moves to the right with speed 1 until time $S<1-x$, where $S$ is uniformly distributed on $(x, 1)$ when starting at $x$. When $S$ occurs the particle jumps to $\{1\}$ if $X\left(S_{-}\right) \epsilon$ $\bigcup_{m=1}^{\infty}\left[2^{-2 m}, 2^{-2 m+1}\right)$ and to $\{2\}$ if $X(S-) \in \bigcup_{m=0}^{\infty}\left[2^{-2 m-1}, 2^{-2 m}\right)$. It is easy to check that this is a Hunt process since $S$ is not an accessible stopping time.

Now let $T$ be the first hit of $A=\left\{2^{-n}, n \geq 1\right\}$ and let $L^{t}$ be generated by $T$ as in Lemma 3.3. Then for $s>0$ if $u$ is sufficiently small

$$
D_{n}(0, u, s,\{1\})= \begin{cases}1 & \text { if } u \in \bigcup_{m=1}^{\infty}\left[2^{-2 m}, 2^{-2 m+1}\right), \\ 0 & \text { otherwise }\end{cases}
$$

and

$$
Q(0, u, s,\{1\})= \begin{cases}2 / 3 & \text { if } u \in \bigcup_{m=1}^{\infty}\left[2^{-2 m}, 2^{-2 m+1}\right), \\ 1 / 3 & \text { otherwise. }\end{cases}
$$

Hence neither $D(0, s,\{1\})$ nor $Q(0, s,\{1\})$ can exist. It is clear, of course, that $X_{L^{t}}$ and $X_{L^{t}}$ equal zero with probability zero, so this example is consistent with our results above.

This process is not "typical" in the sense that $X_{L_{t}}$ and $X_{L^{t}-}$ are not regular with respect to $T$. However, it would seem the nonexistence of $D(0, s, \cdot)$ and $Q(0, s,$.$) may be typical in the sense that starting at \{0\}$ paths visit at arbitrarily small times states with widely differing "last exit distributions" $Q(x, \cdot, \cdot)$ and $D(x, \cdot, \cdot)$.

\section{REFERENCES}

1. R. M. Blumenthal and R. K. Getoor, Markov processes and potential theory, Pure and Appl. Math., vol. 29, Academic Press, New York, 1968. MR 41 \#9348.

2. - A theorem on stopping times, Ann. Math. Statist. 35 (1964), 1348-1350. MR $29 \# 6540$.

3. K. L. Chung and J. L. Doob, Fields, optionality and measurability, Amer. J. Math. 87 (1965), 397-424. MR $35 \# 4972$. 
4. J. L. Doob, Compactification of the discrete state space of a Markov process, $\mathrm{Z}$. Wahrscheinlichkeitstheorie und Verw. Gebiete 10 (1968), 236-25i. MR 38 \# 2842.

5. P. A. Meyer, Probability and potentials, Blaisdell, Waltham, Mass., 1966. MR 34 \# 5119.

6. - Séminaire de probabilités. V, Springer-Verlag, Berlin, 1971, pp. 270-274.

7. P. A. Meyer, R. T. Smythe and J. L. Walsh, Birth and death of Markov processes, Proc. Sixth Berkely Sympos., vol. 3, Univ. of California, Berkely, Calif., 1973, pp. 295-305.

8. A. O. P ittenger, Last exit times and the $Q$-matrices of Marknv chains, $\mathrm{Z}$. Wahrscheinlichkeitstheorie und Verw. Gebiete 20 (1971), 143-162.

9. A. O. Pittenger and C. T. Shih, Coterminal families and the strong Markov property, Bull. Amer. Math. Soc. 78 (1972), 439-443.

10. C. T. Shih, On extending potential theory to all strong Markov processes, Ann. Inst. Fourier (Grenoble) 20 (1970), fasc. 1, 303-315. MR $44 \# 6040$.

DEPARTMENT OF MATHEMATICS, UNIVERSITY OF MICHIGAN, ANN ARBOR, MICHIGAN 48104 (Current address of C. T. Shih)

Current address (A. O. Pittenger): Department of Mathematics, University of Maryland, Baltimore, Maryland 21228 